\title{
DIMINISHING CHOQUET HESITANT 2-TUPLE LINGUISTIC AGGREGATION OPERATOR FOR MULTIPLE ATTRIBUTES GROUP DECISION MAKING
}

\author{
ISMAT BEG ${ }^{1 *}$, RAJA NOSHAD JAMIL ${ }^{2}$ AND TABASAM RASHID ${ }^{2}$ \\ ${ }^{1}$ Lahore School of Economics, Lahore-53200, Pakistan \\ ${ }^{2}$ University of Management and Technology, Lahore-54770, Pakistan \\ ${ }^{*}$ Corresponding author: ibeg@lahoreschool.edu.pk
}

\begin{abstract}
In this article, we develop a diminishing hesitant 2-tuple averaging operator (DH2TA) for hesitant 2-tuple linguistic arguments. DH2TA work in the way that it aggregate all hesitant 2-tuple linguistic elements and during the aggregation process it also controls the hesitation in translation of the resultant aggregated linguistic term. We develop a scalar product for hesitant 2-tuple linguistic elements and based on the scalar product a weighted diminishing hesitant 2-tuple averaging operator (DWH2TA) is introduced. Moreover, combining Choquet integral with hesitant 2-tuple linguistic information, the diminishing Chouqet hesitant 2-tuple average operator (DCH2TA) is defined. The proposed operators higher reflect the correlations among the elements. After investigating the properties of these operators, a multiple attribute decision making method based on DCH2TA operator is proposed. Finally, an example is given to illustrate the significance and usefulness of proposed method.
\end{abstract}

\section{INTRODUCTION}

Different procedures wherein problems that manage indefinite and vague data mostly involves the vulnerability of their definition structures. Utilizing numerical modelling to represent such indeterminate data would not be reliably adequate. In these conditions wherein the vulnerability would not be of probabilistic

Received 2018-10-17; accepted 2018-11-26; published 2019-01-04.

2010 Mathematics Subject Classification. 91B06; 94D05; 90B50; 91B10; 46S40; 03E72.

Key words and phrases. hesitant 2-tuple model; aggregation operator; choquet integral; multiple attribute group decision making; supply chain management.

(C)2019 Authors retain the copyrights of their papers, and all open access articles are distributed under the terms of the Creative Commons Attribution License. 
nature and the capacities are unclear, it is hard to give numerical specific information. Usually the decision makers that take an interest in this kind of issues utilize linguistic descriptors to particular their evaluation and identified with the uncertain potential they have concerning the issues $[38,40]$. Therefore, the utilization of linguistic demonstration in problems managing non-probabilistic instability shows up justification and has made effective result in particular fields, for example: situation realization [35], decision models [6,9,49,64], information retrieval [25,30], risk evaluation [18], engineering analysis [39, 40], sensory evaluation [10,36], performance appraisal [1,2], data mining [27], social alternative [19] and waste management [51]. These accomplishments have not been possible without systems to complete the improvement of computing with words (CW) [34] that implies the utilization of linguistic knowledge. The accompanying algorithm indicates how these translations to be functional.

\section{Algorithm 1.1.}

Step 1. Input data in the form of linguistic terms or 2-tuple linguistic terms

Step 2. Translation into equivalent numeric value

Step 3. Manipulation

Step 4. Retranslation into linguistic terms / 2-tuple linguistic terms accordingly

Step 5. Output data

These ideas for CW have an edge on probability theory [26,33], the uncertainty models, in these problems are alternatively involving the imprecision and vagueness of the linguistic descriptors. For this reason other tools as fuzzy logic [71] and the fuzzy linguistic process [72] used specific computational models for CW, for instance:

- The linguistic computational model created on membership functions such as [13,37] these models based on the fuzzy linguistic approach and makes the computations instantly on the membership function of the linguistic terms by way of utilizing the extension principle [16,31].

- Foundation of the linguistic symbolic computational models are on ordinal scales [65]. These models represent the understanding in keeping with the fuzzy linguistic technique and makes use of the ordered structure of the linguistic term set to achieve symbolic computations in such ordered linguistic scales. Equivalent tactics founded on this mode of computing has been discussed in [14,62]. It notable that this mannequin has been frequently applied to decision making practices due to its easy adaptation and effortlessness for decision makers [65].

Linguistic models seek after the computational plan showed by means of Yager in [66,68] can be seen in general algorithm 1.1, that features out the significance of the interpretation and translation approaches in $\mathrm{CW}$ and likewise Mendel and Wu [42] highlighted similar techniques in computing with perceptions - In the article author discussed that firstly, taking data linguistically and translates into a computing 
tool for manipulative structure. In the second stage, incorporates taking the outcomes from the control, computing device, arrange and change them into linguistic information as an approach to be reasonable by method for individuals that is without uncertainty, one of the essential desire of CW [42]. These linguistic computational units present a most important weak point, in view that they carried out the translation step as an approximation method to precise the outcome in the usual expression area (initial term set) scary a lack of accuracy [23]. To obstruct such inaccuracy in the translation step was once offered the 2-tuple linguistic computational model $[22,41]$. It is a typical mannequin that broadens the utilization of records adjusting the fuzzy linguistic strategy representation with including a parameter with essential linguistic representation as an approach to show signs of improvement exactness of the linguistic calculations after the re-interpretation step, holding the $\mathrm{CW}$ plan stated in algorithm 1.1 and the work out the capacity of the result.

In recent times, numerous aggregation operators have been produced for the 2-tuple linguistic model to assess diverse decision making issues [59]. Herrera and Martínez [22] proposed the 2-tuple arithmetic weighted averaging operator, the 2-tuple ordered weighted averaging operator and the extended 2-tuple weighted averaging operator. Xu et al. [63] developed the extended geometric mean operator, the extended arithmetic averaging operator, the extended ordered weighted averaging operator and the extended ordered weighted geometric operator . Jiang and Fan [28], proposed the 2-tuple ordered weighted averaging operator and the 2-tuple ordered weighted geometric operator. The extended 2-tuple ordered weighted averaging operator was proposed in [73]. The extended 2-tuple weighted geometric operator and the extended 2-tuple ordered weighted geometric operator have been developed in [60]. Herrera et al. [24] proposed an unbalanced linguistic computational model that helpful for calculating the 2-tuple fuzzy linguistic computational model to achieve processes of evaluating words for unbalanced term sets in an accurate mode without loss of information. Furthermore, Dong et al. [15] proposed a consistency improving model which preserves the utmost original knowledge and preferences in the process of improving consistency and it also guarantees that the elements in the optimal adjusted unbalanced linguistic preference relation are all simple unbalanced linguistic terms.

Aggregation operators examined over, the attributes are thought to be autonomous of each other, which are differentiated by an independent axiom $[43,44,57]$. But in the real decision making practice, the characteristics of the problem are often dependent or correlated to each other. Choquet integral [11] is one of the valuable instrument to build up the model an issue, which utilize the properties as between reliance or connection to each other. Choquet integral has examined and connected all the basic properties of the decision making problems $[32,50,67,69]$. Yager [67] proposed the induced Choquet ordered averaging operator to aggregate a group of real arguments while in [69], Yager combined the intuitionistic fuzzy sets with Choquet integral. The intuitionistic fuzzy Choquet integral operator obtained in [9]. Tan and Chen [53] developed 
the induced Choquet ordered averaging operator. Xu [64] proposed the intuitionistic fuzzy correlated averaging operator, the intuitionistic fuzzy correlated geometric operator, the interval-valued intuitionistic fuzzy correlated averaging operator and the interval-valued intuitionistic fuzzy correlated geometric operator to aggregate the intuitionistic fuzzy information and the interval-valued intuitionistic fuzzy information. Beg and Rashid [4] used Choquet integral, for selection of bike when the criteria include interactions among each others. Yang and Zhiping [70] proposed, 2-tuple correlated averaging operator, the 2-tuple correlated geometric operator and the generalized 2-tuple correlated averaging operator combined with Choquet integral. Joshi et al. [29] developed novel hesitant probabilistic fuzzy linguistic ordered weighted averaging and hesitant probabilistic fuzzy linguistic ordered weighted geometric aggregation operators for ill structured and complex decision making problems.

Torra [55] discussed that hesitant fuzzy set can deal with the conditions where the assessment of a selection under each and every criterion is represented by several feasible values, not by a margin of error, or some probability distribution on the possible values. For instance, decision maker gives the membership value of $x$ into $A$, and so they wish to assign $0.23,0.26$ and 0.31 , which is a hesitant fuzzy element $\{0.23,0.26,0.31\}$ rather than the interval between 0.23 and 0.31. Use these qualities of the hesitant fuzzy set, Beg and Rashid [5] proposed hesitant 2-tuple linguistic information to take care of marginal error. As we observed in known literature for hesitant sets, the aggregation operators produced more hesitation during aggregation process with respect to given hesitant elements [74]. Due to this we develop new operational laws for hesitant 2-tuple. These operational laws reduce the hesitation during aggregate process. We used these operational laws to develop a diminishing hesitant 2-tuple averaging operator (DH2TA) for hesitant 2-tuple linguistic arguments. DH2TA worked in two ways, firstly it aggregated all hesitant 2-tuple linguistic elements and secondly it also reduces the hesitation in resultant 2-tuple linguistic term.

In this article, to check applicability of our method we apply it to supply chain management area. In today globalization era, a suitable supplier selection is a core issue of supply chain management that effect the overall performance as without efficient suppliers it is impossible to produce low-cost and high quality products $[3,56]$. Especially for organizations that spend a high level of their business income on parts and material supplies and whose material costs is a large part of aggregate costs. An organized and transparent approach regarding the choice of supplier is essential for these organizations. Supplier selection is a procedure by which suppliers are assessed, evaluated, and then selected to become a part of the company's supply chain [8]. To overcome the supply chain risk, reduced the production cost, optimize inventory levels and the end profitability are major targets of supply chain management $[12,21]$. There exist some well known method for supplier section for instant, matrix approach [20], vendor performance matrix approach [52], vendor profile analysis [54], analytic hierarchy process (AHP) [47,48] and multiple objective programming (MOP) [17]. 
In this paper, we use the notion of hesitant 2-tuple linguistic information which was proposed by Beg and Rashid [5] to develop a diminishing hesitant 2-tuple averaging operator (DH2TA) for hesitant 2-tuple linguistic arguments. The rest of the paper is structured as follows: some basic concepts are presented to understand our proposal in section 2 . In section 3 we propose some definition which is ranking the hesitant 2 tuple linguistic information In Section 4, we define diminishing hesitant 2-tuple averaging (DH2TA) operator, and discussed some properties of DH2TA. In Section 5, we merge Choquet integral with the operator DH2TA and developed a new operator diminishing Choquet hesitant 2-tuple average operator (DCH2TA) and also discussed different properties of DCH2TA. The multiple attribute decision making method based on DCH2TA is proposed in Section 6. In Section 7, a numerical example is given to illustrate the developed approach and to demonstrate its feasibility and practicality. Concluding remarks are given in last section.

\section{Hesitant Fuzzy Sets}

Some important preliminary concepts are given in this section to understand our proposed aggregation operators.

Hesitant fuzzy set was defined by Torra [55] to match the vagueness of real life, when some one is hesitant about membership value.

Definition 2.1. [55] For a reference set $X$. The hesitant fuzzy set on $X$ is defined by function that will give a subset of $[0,1]$ when applied to $X$.

To be easily understood, Xia et al. [61] expressed the HFS by a mathematical symbol:

$$
E=\left\{<x, h_{E}(x)>\mid x \in X\right\}
$$

where $h_{E}(x)$ is set of values form $[0,1]$, known as the possible membership degrees of $x$ to set $E$. Also $h=h_{E}(x)$ is called hesitant fuzzy element (HFE).

To find order between two HFEs, Xia et al. [61] defined score function as follow:

Definition 2.2. [61] Let $e$ be a HFE and $h \in e$ then score function " $S$ " of $e$ is

$$
S(e)=\frac{1}{n(e)} \sum_{i=1}^{n(e)} h_{i}
$$

where $n(e)$ be total number of elements in $e$.

Let $e_{1}$ and $e_{2}$ be two HFEs then,

$$
\text { if } S\left(e_{1}\right)<S\left(e_{2}\right) \text { then } e_{1} \prec e_{2}
$$

and

$$
\text { if } S\left(e_{1}\right)=S\left(e_{2}\right) \text { then } e_{1} \approx e_{2}
$$


Let $e, e_{1}$ and $e_{2}$ be elements of a hesitant fuzzy set $A$ then following basic operations are introduced by Xia et al. [61]:

(1) $e^{\alpha}=\cup_{h \in e}\left\{h^{\alpha}\right\}, \alpha>0$.

(2) $\alpha e=\cup_{h \in e}\left\{1-(1-h)^{\alpha}\right\}, \alpha>0$.

(3) $h_{1} \oplus h_{2}=\cup_{h_{1} \in e_{1}, h_{2} \in e_{2}}\left\{h_{1}+h_{2}-h_{1} h_{2}\right\}$.

(4) $h_{1} \otimes h_{2}=\cup_{h_{1} \in e_{1}, h_{2} \in e_{2}}\left\{h_{1} h_{2}\right\}$.

Next we study concise review of 2-tuple linguistic information and some important basic concepts which are necessary to develop the aggregation operator for hesitant 2-tuple linguistic information.

Assume that $L=\left\{l_{i} \mid i=2 n+1, \forall n \in N\right\}$ where $N$ be the set of natural number and $l_{i}$ be representation of a possible value for linguistic variable. The set $L$ have the following properties by [22]:

P 1 . The set $L$ must be ordered: $l_{i} \geq l_{j}$ if $i \geq j$,

$\mathrm{P} 2$. The maximum of any two linguistic terms is $\max \left(l_{i}, l_{j}\right)=l_{i}$ if $l_{i} \geq l_{j}$,

$\mathrm{P}$ 3. The minimum of any two linguistic terms is $\min \left(l_{i}, l_{j}\right)=l_{i}$ if $l_{i} \leq l_{j}$.

The cardinality of the set $L$ must be low enough that is not to impose unnecessary precision for users and it should be rich enough to allow discrimination of the performance of the individual criteria in the limited number of ranking. Psychologist [45] recommend the use of $7 \pm 2$ labels. Due to this point of view, a linguistic term set, $L$ with seven labels can be defined as follows:

$L=\left\{l_{0}=\right.$ extremely unattractive $(E U), l_{1}=$ fairly unattractive $(F U), l_{2}=$ unattractive $(U), l_{3}=$ normal $(N), l_{4}=\operatorname{attractive}(A), l_{5}=$ fairly attractive $(F A), l_{6}=$ extremely attractive $\left.(E A)\right\}$.

In the literature different models have been recommended for processing of linguistic information. In this paper, we have implemented 2-tuple linguistic representation model, which is based on symbolic translation [22]. Symbolic translation is defined as follow:

Definition 2.3. [22] Let $L=\left\{l_{0}, l_{1}, \ldots, l_{g}\right\}$ be the set of linguistic terms, $\delta_{i} \in[0, g]$ for any $i \in\{0,1, \ldots, g\}$, $j=\operatorname{round}\left(\delta_{i}\right)$ and $\varsigma_{j}=\delta_{i}-j \Longrightarrow \varsigma_{j} \in[-0.5,0.5)$, then $\varsigma_{j}$ is called the value of the symbolic translation. Where round $\left(\delta_{i}\right)$ is the usual round operation on label index of set $L$.

Definition 2.4. [22] Let $L=\left\{l_{0}, l_{1}, l_{2}, \ldots, l_{g}\right\}$ be the set of linguistic terms set and $\delta_{i}$ be the number representing the aggregation result of symbolic operation. The function $\triangle$ used to obtain the 2-tuple linguistic information equivalent to $\delta_{i}$ is defined as:

$$
\begin{gathered}
\triangle \quad: \quad[0, g] \longrightarrow L \times[-0.5,0.5), \\
\triangle\left(\delta_{i}\right)=\left(l_{j}, \varsigma_{j}\right) \text { with } \begin{cases}l_{j} & j=\operatorname{round}\left(\delta_{i}\right) \\
\varsigma_{j}=\delta_{i}-j & \varsigma_{j} \in[-0.5,0.5)\end{cases}
\end{gathered}
$$




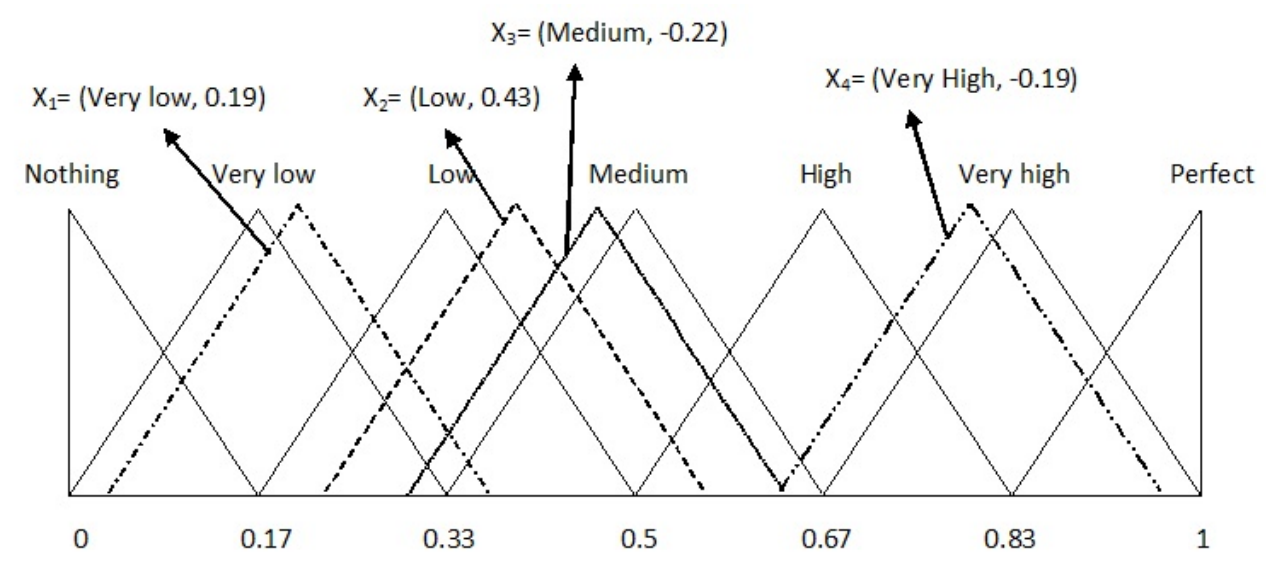

Figure 1. Structure of different 2-tuple linguistic elements

Inverse function of $\triangle$ is always exist and denoted by $\Delta^{-1}$.

$$
\begin{aligned}
\triangle^{-1} & : \quad L \times[-0.5,0.5) \longrightarrow[0, g], \\
\triangle^{-1}\left(l_{j}, \varsigma_{j}\right) & =\varsigma_{j}+j=\delta_{i}
\end{aligned}
$$

Example 2.1. Suppose we have different 2 - tuple elements $x_{1}=($ Nothing, 0.19$), x_{2}=($ Low, 0.43), $x_{3}=($ Medium,-0.22$)$ and $x_{4}=($ Very high,-0.19$)$ then the structure of these elements is described in Figure 1.

Definition 2.5. [22] Let $\left(l_{i}, \varsigma_{i}\right)$ and $\left(l_{j}, \varsigma_{j}\right)$ be two 2-tuple linguistic elements, then order between them is according to an ordinary lexicographic order:

(1) If $i<j$ then $\left(l_{i}, \varsigma_{i}\right)<\left(l_{j}, \varsigma_{j}\right)$,

(2) If $i=j$ then

- if $\varsigma_{i}<\varsigma_{j}$ then $\left(l_{i}, \varsigma_{i}\right)<\left(l_{j}, \varsigma_{j}\right)$

- if $\varsigma_{i}=\varsigma_{j}$ then $\left(l_{i}, \varsigma_{i}\right)=\left(l_{j}, \varsigma_{j}\right)$

Definition 2.6. [58] A fuzzy measure $\alpha$ on the set $X$ is a set function $\alpha: P(X) \rightarrow[0,1]$ satisfying the following conditions:

(1) $\alpha(\emptyset)=0, \alpha(X)=1$;

(2) If $B \subseteq C \Rightarrow \alpha(B) \leq \alpha(C), \forall B, C \subseteq X$;

(3) $\alpha(B \cup C)=\alpha(B)+\alpha(C)+\lambda \alpha(B) \alpha(C) \forall B, C \subseteq X$ and $B \cap C=\emptyset$, where $\lambda \in(-1,+\infty)$. 
By parameter $\lambda$ the interaction between criteria can be represented.

$\bigcup_{i=1}^{n} x_{i}=X$ for a finite set $X$. The $\lambda$ - fuzzy measure $\alpha$ satisfied the following equation

$$
\alpha(X)=\alpha\left(\bigcup_{i=1}^{n} x_{i}\right)=\left\{\begin{array}{lr}
\frac{1}{\lambda}\left\{\prod_{i=1}^{n}\left(1+\lambda \alpha\left(x_{i}\right)\right)-1\right\} & \text { if } \lambda \neq 0 \\
\sum_{i=1}^{n} \alpha\left(x_{i}\right) & \lambda=0
\end{array}\right.
$$

where $x_{i} \cap x_{j}=\emptyset$ for all $i, j=1,2, \ldots, n$ and $i \neq j$. The number $\alpha\left(x_{i}\right)$ for a subset with a single element $\left\{x_{i}\right\}$ is called a fuzzy density.

$$
\alpha(A)=\left\{\begin{array}{lr}
\frac{1}{\lambda}\left\{\prod_{i=1}^{n}\left(1+\lambda \alpha\left(x_{i}\right)\right)-1\right\} & \text { if } \lambda \neq 0 \\
\sum_{i=1}^{n} \alpha\left(x_{i}\right) & \lambda=0
\end{array}\right.
$$

Based on above equation, the value of $\lambda$ can be find from the following equation, if $\alpha(X)=1$ then,

$$
1=\frac{1}{\lambda}\left\{\prod_{i=1}^{n}\left(1+\lambda \alpha\left(x_{i}\right)\right)-1\right\}
$$

In the above definition, if $\lambda=0$, then the third condition reduces to the axiom of the additive measure i.e. $\alpha(B \cup C)=\alpha(B)+\alpha(C) \forall B, C \subseteq X$ and $B \cap C=\emptyset$

If the elements of $B$ are independent, then $\alpha(B)=\sum_{x_{i} \in B} \alpha\left(x_{i}\right) \forall B \subseteq X$.

\section{Hesitant 2-tuple Linguistic Information}

Hesitant 2-tuple linguistic information model is introduced by Beg and Rashid [5] to manage the conditions in which information described is in linguistic term and decision maker has some hesitation to decide its possible linguistic translations.

Let $X$ be a universe of discourse and $L=\left\{l_{0}, l_{1}, l_{2}, \ldots, l_{g}\right\}$ be the linguistic term set then a hesitant 2-tuple linguistic term set in $X$ is an expression $E=\{(x, h(x)): x \in X\}$, where $h(x)=\left(l_{i}, \varsigma_{i, j}\right)$ be the hesitant linguistic information by mean of 2 -tuple, $\varsigma_{i, j}$ is finite subset of $[-0.5,0.5)$ which represent the possible translations of $l_{i}$ and $j$ be the cardinality of $\varsigma_{i, j}$ and $i \in\{0,1,2, \ldots, g\}$

Definition 3.1. Let $h(x)=\left(l_{i}, \varsigma_{i, j}\right)$ be a H2TLE then score function $S$ of $h(x)$ is

$$
S(h(x))=\frac{1}{j} \sum_{\gamma \in \varsigma_{i, j}} \gamma \text { where } j \text { is the cardinality of } \varsigma_{i, j}
$$

To find order between two H2TLE we use the score function defined in definition 3.1

Definition 3.2. Let $h_{1}(x)=\left(l_{i}, \varsigma_{i, j}\right)$ and $h_{2}(x)=\left(l_{k}, \varsigma_{k, p}\right)$ be two H2TLEs, then order between them is according to an ordinary lexicographic order: 
(1) If $i<k$ then $h_{1}(x) \prec h_{2}(x)$,

(2) If $i=k$ and

- $S\left(h_{1}(x)\right)<S\left(h_{2}(x)\right)$ then $h_{1}(x) \prec h_{2}(x)$

- $S\left(h_{1}(x)\right)=S\left(h_{2}(x)\right)$ then $h_{1}(x)=h_{2}(x)$

Definition 3.3. Let $\left(l_{i_{p}}, \varsigma_{i_{p}, j_{p}}\right)$ be $k$ H2TLEs. If $k \in \mathbb{N}, i_{p} \in\{0,1,2, \ldots, g\}$ and $j_{p}$ be cardinality of $\varsigma_{i_{p}, j_{p}}$, then $\min _{p=1}^{k}\left(l_{i_{p}}, \varsigma_{i_{p}, j_{p}}\right)$ is defined as follow:

(1) If all $\left(l_{i_{p}}, \varsigma_{i_{p}, j_{p}}\right)$ are different due to different $i_{p}$ then, let $\min _{p=1}^{k}\left(i_{p}\right)=i \in\{0,1,2, \ldots, g\}$. If $\varsigma_{i, j}$ be represent the translations of $l_{i}$ and $j$ be the cardinality of $\varsigma_{i, j}$, then by definition 3.2

$$
\min _{p=1}^{k}\left(l_{i_{p}}, \varsigma_{i_{p}, j_{p}}\right)=\left(l_{i}, \varsigma_{i, j}\right)
$$

(2) If $i_{p}=i$ but $\varsigma_{i_{p}, j_{p}}$ are different for each $\left(l_{i_{p}}, \varsigma_{i_{p}, j_{p}}\right)$. If $\varsigma_{i, j}$ is represent the translations of $l_{i_{p}}=l_{i}$ with $S\left(l_{i_{p}}, \varsigma_{i_{p}, j_{p}}\right)=\min _{p=1}^{k}\left(\frac{1}{j_{p}} \sum_{\gamma \in \varsigma_{i_{p}, j_{p}}} \gamma\right)$ then

$$
\min _{p=1}^{k}\left(l_{i_{p}}, \varsigma_{i_{p}, j_{p}}\right)=\left(l_{i}, \varsigma_{i, j}\right)
$$

(3) If all $\left(l_{i_{p}}, \varsigma_{i_{p}}, j_{p}\right)$ are equal, such that $i_{p}=i$ and $\varsigma_{i_{p}, j_{p}}=\varsigma_{i, j}$, then by definition 3.2

$$
\min _{p=1}^{k}\left(l_{i_{p}}, \varsigma_{i_{p}, j_{p}}\right)=\left(l_{i}, \varsigma_{i, j}\right)
$$

(4) If all $\left(l_{i_{p}}, \varsigma_{i_{p}, j_{p}}\right)$ H2TLEs are equal by definition 3.2 but still there is some possibility exist that $\varsigma_{i_{p}, j_{p}}$ are different, but score are same then $\min _{p=1}^{k}\left(l_{i_{p}}, \varsigma_{i_{p}, j_{p}}\right)$ is one with maximum hesitation.

Definition 3.4. Let $\left(l_{i_{p}}, \varsigma_{i_{p}, j_{p}}\right)$ be $k$ H2TLEs. If $k \in \mathbb{N}, i_{p} \in\{0,1,2, \ldots, g\}$ and $j_{p}$ be cardinality of $\varsigma_{i_{p}, j_{p}}$, then $\max _{p=1}^{k}\left(l_{i_{p}}, \varsigma_{i_{p}, j_{p}}\right)$ is defined as follow:

(1) If all $\left(l_{i_{p}}, \varsigma_{i_{p}, j_{p}}\right)$ are different due to different $i_{p}$ then, let $\max _{p=1}^{k}\left(i_{p}\right)=i \in\{0,1,2, \ldots, g\}$. If $\varsigma_{i, j}$ be represent the translations of $l_{i}$ and $j$ be the cardinality of $\varsigma_{i, j}$, then by definition 3.2

$$
\max _{p=1}^{k}\left(l_{i_{p}}, \varsigma_{i_{p}, j_{p}}\right)=\left(l_{i}, \varsigma_{i, j}\right)
$$

(2) If $i_{p}=i$ but $\varsigma_{i_{p}, j_{p}}$ are different for each $\left(l_{i_{p}}, \varsigma_{i_{p}, j_{p}}\right)$. If $\varsigma_{i, j}$ is represent the translations of $l_{i_{p}}=l_{i}$ with $S\left(l_{i_{p}}, \varsigma_{i_{p}, j_{p}}\right)=\max _{p=1}^{k}\left(\frac{1}{j_{p}} \sum_{\gamma \in \varsigma_{i_{p}, j_{p}}} \gamma\right)$ then

$$
\max _{p=1}^{k}\left(l_{i_{p}}, \varsigma_{i_{p}, j_{p}}\right)=\left(l_{i}, \varsigma_{i, j}\right)
$$

(3) If all $\left(l_{i_{p}}, \varsigma_{i_{p}, j_{p}}\right)$ are equal, such that $i_{p}=i$ and $\varsigma_{i_{p}, j_{p}}=\varsigma_{i, j}$, then by definition 3.2

$$
\max _{p=1}^{k}\left(l_{i_{p}}, \varsigma_{i_{p}, j_{p}}\right)=\left(l_{i}, \varsigma_{i, j}\right)
$$


(4) If all $\left(l_{i_{p}}, \varsigma_{i_{p}, j_{p}}\right)$ H2TLEs are equal by definition 3.2 but still there is some possibility exist that $\varsigma_{i_{p}, j_{p}}$ are different, but score are same then $\max _{p=1}^{k}\left(l_{i_{p}}, \varsigma_{i_{p}, j_{p}}\right)$ is one with minimum hesitation.

Definition 3.5. If $\left(l_{k_{1}}, \varsigma_{k_{1}, j_{1}}\right)$ is a hesitant 2-tuple linguistic element, $g$ be the upper limit of the linguistic term set and $\lambda \geq 0$ is any scalar. Then the scalar product for H2TLE is defined as follows:

$$
\lambda\left(l_{k_{1}}, \varsigma_{k_{1}, j_{1}}\right)=\left(\lambda l_{k_{1}}, \lambda \varsigma_{k_{1}, j_{1}}\right)=\left(l_{i}, \varsigma_{i, j}\right)
$$

$\left(l_{i}, \varsigma_{i, j}\right)$ is calculated as follow

$$
\begin{aligned}
\text { Let } \beta_{1} & =\left\{\mu \mid \mu=\left((g+1)\left(1-\left(1-\frac{g+\gamma_{k_{1}, j_{1}}}{g+1}\right)^{\lambda}\right) \forall \gamma_{k_{1}, j_{1}} \in \varsigma_{k_{1}, j_{1}}\right\},\right. \\
\text { then } \beta_{2} & =\bigcup\left\{\theta_{1} \mid \theta_{1}=\operatorname{round}(\mu), \forall \mu \in \beta_{1}\right\}, i=\frac{\left(\sum_{\theta_{1} \in \beta_{2}} \theta_{1}+k_{1}\right)}{\left|\beta_{2}\right|+1},\left|\beta_{2}\right|
\end{aligned}
$$

be cardinality of $\beta_{2} \varsigma_{i_{p}, j_{p}}=\left\{\theta_{2} \mid \theta_{2}=\operatorname{different}\left(\mu-\theta_{1}\right)\right\}, j_{p}$ be cardinality of each $\varsigma_{i_{p}, j_{p}}$

$$
\begin{aligned}
\varsigma_{i, j}= & \left\{x \mid x \in \varsigma_{1} \cap \varsigma_{2}\right\} \text { for all } r_{q} \in \eta=\bigcup_{p=1}^{k} \varsigma_{i_{p}, j_{p}}, \text { while } \\
\varsigma_{1}= & \bigcup_{q=1}^{|\eta|}\left\{\min \left(r_{q}, \max \left(\begin{array}{c}
\min \left(\bigcup_{p=1}^{k}\left\{\max \left(\varsigma_{i_{p}, j_{p}}\right)\right\}\right), \\
\max \left(\bigcup_{p=1}^{k} \min \left\{\left(\varsigma_{i_{p}, j_{p}}\right)\right\}\right)
\end{array}\right)\right\},\right. \\
\text { and } \varsigma_{2}= & \bigcup_{q=1}^{|\eta|}\left\{\max \left(r_{q}, \min \left(\begin{array}{c}
\min \left(\bigcup_{p=1}^{k}\left\{\max \left(\varsigma_{i_{p}, j_{p}}\right)\right\}\right), \\
\max \left(\bigcup_{p=1}^{k}\left\{\min \left(\varsigma_{i_{p}, j_{p}}\right)\right\}\right)
\end{array}\right)\right\},\right.
\end{aligned}
$$

where round $(*)$ is usual round operation.

\section{Diminishing Hesitant 2-tuple Averaging Operator}

Beg and Rashid [5] discussed a model which is characterized by a linguistic term and its possible symbolic translations. This model is more suitable for dealing with fuzziness and uncertainty than the 2-tuple linguistic arguments. In this section, we defined an operator for the hesitant 2-tuple linguistic elements to handle the situation, where experts face some hesitation to present its possible linguistic translations.

Definition 4.1. If $h_{1}=\left(l_{i_{1}}, \varsigma_{i_{1}, j_{1}}\right), h_{2}=\left(l_{i_{2}}, \varsigma_{i_{2}, j_{2}}\right), \ldots, h_{k}=\left(l_{i_{k}}, \varsigma_{i_{k}, j_{k}}\right)$ are $k$, 2-tuple hesitant linguistic terms where $j_{p}$ is the cardinality of $\varsigma_{i_{p}, j_{p}}$, then diminishing hesitant 2-tuple averaging operator (DH2TA) is 
defined as

$$
\begin{aligned}
& \operatorname{DH} 2 T A\left(\left(l_{i_{1}}, \varsigma_{i_{1}, j_{1}}\right),\left(l_{i_{2}}, \varsigma_{i_{2}, j_{2}}\right), \ldots,\left(l_{i_{k}}, \varsigma_{i_{k}, j_{k}}\right)\right) \\
& =\left(l_{i}, \varsigma_{i, j}\right) \text { where } i=\operatorname{round}\left(\frac{i_{1}+i_{2}+\ldots+i_{k}}{k}\right) \text { and } \\
& \varsigma_{i, j}=\left\{x \mid x \in \varsigma_{1} \cap \varsigma_{2}\right\} \text { for all } r_{q} \in \eta=\bigcup_{p=1}^{k} \varsigma_{i_{p}, j_{p}} \text { we have } \\
& \varsigma_{1}=\bigcup_{q=1}^{|\eta|}\left\{\min \left(r_{q}, \max \left(\begin{array}{c}
\min \left(\bigcup_{p=1}^{k}\left\{\max \left(\varsigma_{i_{p}, j_{p}}\right)\right\}\right), \\
\max \left(\bigcup_{p=1}^{k}\left\{\min \left(\varsigma_{i_{p}, j_{p}}\right)\right\}\right.
\end{array}\right)\right)\right\} \\
& \operatorname{and} \varsigma_{2}=\bigcup_{q=1}^{|\eta|}\left\{\max \left(r_{q}, \min \left(\begin{array}{c}
\min \left(\bigcup_{p=1}^{k}\left\{\max \left(\varsigma_{i_{p}, j_{p}}\right)\right\}\right), \\
\max \left(\bigcup_{p=1}^{k}\left\{\min \left(\varsigma_{i_{p}, j_{p}}\right)\right\}\right)
\end{array}\right)\right\}\right.
\end{aligned}
$$

where round $(*)$ be the round function and $|\eta|$ be the cardinality of $\eta$.

Example 4.1. Let $h_{1}=\left(l_{2},\{-0.3,-0.25,-0.1,0.0,0.2\}\right), h_{2}=\left(l_{3},\{-0.2,-0.1,0.1\right.$ $, 0.2,0.25\})$ and $h_{3}=\left(l_{3},\{0.1,0.23,0.3\}\right)$ be 2-tuple hesitant linguistic terms, then,

$$
\operatorname{DH} 2 T A\left(h_{1}, h_{2}, h_{3}\right)=\left(l_{3},\{0.1,0.2\}\right)
$$

Theorem 4.1. Let $\left(l_{i_{1}}, \varsigma_{i_{1}, j_{1}}\right),\left(l_{i_{2}}, \varsigma_{i_{2}, j_{2}}\right), \ldots,\left(l_{i_{k}}, \varsigma_{i_{k}, j_{k}}\right)$ be $k$ 2-tuple hesitant linguistic terms, where $j_{1}, j_{2}, \ldots, j_{k}$ be the cardinality of $\varsigma_{i_{1}, j_{1}}, \varsigma_{i_{2}, j_{2}}, \ldots, \varsigma_{i_{k}, j_{k}}$ respectively, $i_{1}, i_{2}, \ldots, i_{k} \in\{0,1,2, \ldots, m\}$ If all $k$ 2-tuple hesitant linguistic terms are equal i.e. $l_{i_{1}}=l_{i_{2}}=\ldots=l_{i_{k}}=l_{i}$ and also $\varsigma_{i_{1}, j_{1}}=\varsigma_{i_{2}, j_{2}}=\ldots=\varsigma_{i_{k}, j_{k}}=\varsigma_{i, j}$ then,

$$
\operatorname{DH} 2 T A\left(\left(l_{i_{1}}, \varsigma_{i_{1}, j_{1}}\right),\left(l_{i_{2}}, \varsigma_{i_{2}, j_{2}}\right), \ldots,\left(l_{i_{k}}, \varsigma_{i_{k}, j_{k}}\right)\right)=\left(l_{i}, \varsigma_{i, j}\right)
$$

Proof. As $l_{i_{1}}=l_{i_{2}}=\ldots=l_{i_{k}}=l_{i}$ and $\varsigma_{i_{1}, j_{1}}=\varsigma_{i_{2}, j_{2}}=\ldots=\varsigma_{i_{k}, j_{k}}=\varsigma_{i, j}$ therefore

$$
\left(\frac{i_{1}+i_{2}+\ldots+i_{k}}{k}\right)=\left(\frac{i+i+\ldots+i}{k}\right)=i .
$$

and

$$
\varsigma_{i, j}=\bigcup_{p=1}^{k} \varsigma_{i_{p}, j_{p}}
$$

Let

$$
\begin{aligned}
& \varepsilon_{1}=\min \left(\bigcup_{p=1}^{k}\left\{\max \left(\varsigma_{i_{p}, j_{p}}\right)\right\}\right), \varepsilon_{2}=\max \left(\bigcup_{p=1}^{k}\left\{\min \left(\varsigma_{i_{p}, j_{p}}\right)\right\}\right), \\
& r_{1}=\max \left(\varepsilon_{1}, \varepsilon_{2}\right) \text { and } r_{2}=\min \left(\varepsilon_{1}, \varepsilon_{2}\right)
\end{aligned}
$$


from equation 4.1 and 4.2 ,

$$
\begin{aligned}
\varsigma_{1} & =\bigcup_{p=1}^{k}\left\{\min \left(r_{p}, r_{1}\right)\right\}=\varsigma_{i, j} \text { and } \varsigma_{2}=\bigcup_{p=1}^{k}\left\{\max \left(r_{p}, r_{2}\right)\right\}=\varsigma_{i, j} \\
& \Longrightarrow \varsigma_{i, j}=\varsigma_{1} \cap \varsigma_{2} \text { where } r_{p} \in \varsigma_{i, j}
\end{aligned}
$$

Hence $\operatorname{DH} 2 T A\left(\left(l_{i_{1}}, \varsigma_{i_{1}, j_{1}}\right),\left(l_{i_{2}}, \varsigma_{i_{2}, j_{2}}\right), \ldots,\left(l_{i_{k}}, \varsigma_{i_{k}, j_{k}}\right)\right)=\left(l_{i}, \varsigma_{i, j}\right)$.

Theorem 4.2. Let $\left(l_{i_{1}}, \varsigma_{i_{1}, j_{1}}\right),\left(l_{i_{2}}, \varsigma_{i_{2}, j_{2}}\right), \ldots,\left(l_{i_{k}}, \varsigma_{i_{k}, j_{k}}\right)$ be $k$, 2-tuple hesitant linguistic terms where $j_{p}$ be the cardinality of $\varsigma_{i_{p}, j_{p}}$ for $i_{p}=0,1,2, \ldots, g, j_{p}=1,2, \ldots, n$ and $p=1,2, \ldots, k$, then

$\min \left(\left(l_{i_{1}}, \varsigma_{i_{1}, j_{1}}\right),\left(l_{i_{2}}, \varsigma_{i_{2}, j_{2}}\right), \ldots,\left(l_{i_{k}}, \varsigma_{i_{k}, j_{k}}\right)\right) \leq D H 2 T A$

$\left(\left(l_{i_{1}}, \varsigma_{i_{1}, j_{1}}\right),\left(l_{i_{2}}, \varsigma_{i_{2}, j_{2}}\right), \ldots,\left(l_{i_{k}}, \varsigma_{i_{k}, j_{k}}\right)\right) \leq \max \left(\left(l_{i_{1}}, \varsigma_{i_{1}, j_{1}}\right),\left(l_{i_{2}}, \varsigma_{i_{2}, j_{2}}\right), \ldots,\left(l_{i_{k}}, \varsigma_{i_{k}, j_{k}}\right)\right)$.

Proof. As each 2-tuple hesitant linguistic term $\left(l_{i_{p}}, \varsigma_{i_{p}, j_{p}}\right)$ consist of two parts such that $l_{i_{p}}$ be the hesitant linguistic information by mean of 2 -tuple and $\varsigma_{i_{p}, j_{p}}$ is a finite subset of $[-0.5,0.5)$ which represent the possible translations of $l_{i_{p}}$, where $j_{p}$ be the cardinality of $\varsigma_{i_{p}, j_{p}}$ and $i_{p} \in\{0,1,2, \ldots, g\}$, then

Case 1. If all $\left(l_{i_{p}}, \varsigma_{i_{p}}, j_{p}\right)$ are different due to different $i_{p}$ then, by definition 4.1

$$
\begin{array}{r}
\operatorname{DH} 2 T A\left(\left(l_{i_{1}}, \varsigma_{i_{1}, j_{1}}\right),\left(l_{i_{2}}, \varsigma_{i_{2}, j_{2}}\right), \ldots,\left(l_{i_{k}}, \varsigma_{i_{k}, j_{k}}\right)\right)=\left(l_{i}, \varsigma_{i, j}\right) \\
i=\operatorname{round}\left(\frac{i_{1}+i_{2}+\ldots+i_{k}}{k}\right) \text { clearly } \min _{p=1}^{k}\left(i_{p}\right) \leq i \leq \max _{p=1}^{k}\left(i_{p}\right)
\end{array}
$$

therefore by definitions $3.2,3.3,3.4$ and 4.1 we have

$$
\begin{aligned}
& \min \left(\left(l_{i_{1}}, \varsigma_{i_{1}, j_{1}}\right),\left(l_{i_{2}}, \varsigma_{i_{2}, j_{2}}\right), \ldots,\left(l_{i_{k}}, \varsigma_{i_{k}, j_{k}}\right)\right) \\
\leq & \operatorname{DH} 2 \mathrm{TA}\left(\left(l_{i_{1}}, \varsigma_{i_{1}, j_{1}}\right),\left(l_{i_{2}}, \varsigma_{i_{2}, j_{2}}\right), \ldots,\left(l_{i_{k}}, \varsigma_{i_{k}, j_{k}}\right)\right) \\
\leq & \max \left(\left(l_{i_{1}}, \varsigma_{i_{1}, j_{1}}\right),\left(l_{i_{2}}, \varsigma_{i_{2}, j_{2}}\right), \ldots,\left(l_{i_{k}}, \varsigma_{i_{k}, j_{k}}\right)\right)
\end{aligned}
$$

Case 2. If $i_{p}=i$ for all $\left(l_{i_{p}}, \varsigma_{i_{p}}, j_{p}\right)$ but $\varsigma_{i_{p}, j_{p}}$ are different for each $p=1,2,3 \ldots, k$, then,

$$
\text { round }\left(\frac{i_{1}+i_{2}+\ldots+i_{k}}{k}\right)=\operatorname{round}\left(\frac{i+i+\ldots+i}{k}\right)=i
$$

Let

$$
\begin{gathered}
\operatorname{DH} 2 T A\left(\left(l_{i_{1}}, \varsigma_{i_{1}, j_{1}}\right),\left(l_{i_{2}}, \varsigma_{i_{2}, j_{2}}\right), \ldots,\left(l_{i_{k}}, \varsigma_{i_{k}, j_{k}}\right)\right)=\left(l_{i}, \varsigma_{i, j}\right), \\
\min \left(\left(l_{i_{1}}, \varsigma_{i_{1}, j_{1}}\right),\left(l_{i_{2}}, \varsigma_{i_{2}, j_{2}}\right), \ldots,\left(l_{i_{k}}, \varsigma_{i_{k}, j_{k}}\right)\right)=\left(l_{i}, \varsigma_{i, j}^{\min }\right),
\end{gathered}
$$

and

$$
\max \left(\left(l_{i_{1}}, \varsigma_{i_{1}, j_{1}}\right),\left(l_{i_{2}}, \varsigma_{i_{2}, j_{2}}\right), \ldots,\left(l_{i_{k}}, \varsigma_{i_{k}, j_{k}}\right)\right)=\left(l_{i}, \varsigma_{i, j}^{\max }\right) .
$$

Consider $S\left(l_{i}, \varsigma_{i, j}\right), S\left(l_{i}, \varsigma_{i, j}^{\min }\right)$ and $S\left(l_{i}, \varsigma_{i, j}^{\max }\right)$ are scores of $\left(l_{i}, \varsigma_{i, j}\right),\left(l_{i}, \varsigma_{i, j}^{\min }\right)$ and $\left(l_{i}, \varsigma_{i, j}^{\max }\right)$ respectively. Then, by definition of 3.2 and 4.1 , 
Case 2.1. Let $\max \left(\varsigma_{i, j}^{\min }\right) \leq \min \left(\varsigma_{i, j}^{\max }\right) \Longrightarrow \max \left(\varsigma_{i, j}^{\min }\right) \leq r \leq \min \left(\varsigma_{i, j}^{\max }\right) \forall r \in \varsigma_{i, j} \Longrightarrow S\left(l_{i}, \varsigma_{i, j}^{\min }\right) \leq S\left(l_{i}, \varsigma_{i, j}\right) \leq$ $S\left(l_{i}, \varsigma_{i, j}^{\max }\right)$. Therefore,

$$
\left(l_{i}, \stackrel{\varsigma_{i, j}}{\min }\right) \leq \operatorname{DH} 2 \mathrm{TA}\left(\left(l_{i_{1}}, \varsigma_{i_{1}, j_{1}}\right),\left(l_{i_{2}}, \varsigma_{i_{2}, j_{2}}\right), \ldots,\left(l_{i_{k}}, \varsigma_{i_{k}, j_{k}}\right)\right) \leq\left(l_{i}, \varsigma_{i, j}^{\max }\right)
$$

Case 2.2. Let $\max \left(\varsigma_{i, j}^{\min }\right) \geq \min \left(\varsigma_{i, j}^{\max }\right) \Longrightarrow \min \left(\varsigma_{i, j}^{\max }\right) \leq r \leq \max \left(\varsigma_{i, j}^{\min }\right) \forall r \in \varsigma_{i, j} \Longrightarrow S\left(l_{i}, \varsigma_{i, j}^{\min }\right) \leq S\left(l_{i}, \varsigma_{i, j}\right) \leq$ $S\left(l_{i}, \varsigma_{i, j}^{\max }\right)$. Therefore,

$$
\left(l_{i}, \varsigma_{i, j}^{\min }\right) \leq \operatorname{DH} 2 T A\left(\left(l_{i_{1}}, \varsigma_{i_{1}, j_{1}}\right),\left(l_{i_{2}}, \varsigma_{i_{2}, j_{2}}\right), \ldots,\left(l_{i_{k}}, \varsigma_{i_{k}, j_{k}}\right)\right) \leq\left(l_{i}, \varsigma_{i, j}^{\max }\right)
$$

Case 2.3. Let $\min \left(\varsigma_{i, j}^{\min }\right) \leq \max \left(\varsigma_{i, j}^{\max }\right) \leq \max \left(\varsigma_{i, j}^{\min }\right) \Longrightarrow \varsigma_{i, j}=\varsigma_{i, j}^{\max } \Longrightarrow S\left(l_{i}, \varsigma_{i, j}^{\max }\right)=S\left(l_{i}, \varsigma_{i, j}\right)$. Therefore,

$$
\operatorname{DH} 2 T A\left(\left(l_{i_{1}}, \varsigma_{i_{1}, j_{1}}\right),\left(l_{i_{2}}, \varsigma_{i_{2}, j_{2}}\right), \ldots,\left(l_{i_{k}}, \varsigma_{i_{k}, j_{k}}\right)\right)=\left(l_{i}, \varsigma_{i, j}^{\max }\right)
$$

Equations 4.3,4.4,4.5,4.6 and 4.7 provide the required result.

Theorem 4.3. If $\left(l_{i_{p}}, \varsigma_{i_{p}, j_{p}}\right) \leq\left(l_{i_{p}^{\prime}}^{\prime}, \varsigma_{i_{p}^{\prime}, j_{p}^{\prime}}^{\prime}\right)$ for $i_{p}, i_{p}^{\prime} \in\{0,1,2, \ldots, g\}$, $j_{p}, j_{p}^{\prime} \in\{1,2, \ldots, n\}$ and $p=1,2, \ldots, k$, then

$$
\begin{aligned}
& \operatorname{DH} 2 T A\left(\left(l_{i_{1}}, \varsigma_{i_{1}, j_{1}}\right),\left(l_{i_{2}}, \varsigma_{i_{2}, j_{2}}\right), \ldots,\left(l_{i_{k}}, \varsigma_{i_{k}, j_{k}}\right)\right) \\
\leq & \operatorname{DH} 2 T A\left(\left(l_{i_{1}}^{\prime}, \varsigma_{i_{1}, j_{1}}^{\prime}\right),\left(l_{i_{2}}^{\prime}, \varsigma_{i_{2}, j_{2}}^{\prime}\right), \ldots,\left(l_{i_{k}}^{\prime}, \varsigma_{i_{k}, j_{k}}^{\prime}\right)\right)
\end{aligned}
$$

Proof. Given that $\left(l_{i_{p}}, \varsigma_{i_{p}, j_{p}}\right) \leq\left(l_{i_{p}}^{\prime}, \varsigma_{i_{p}, j_{p}}^{\prime}\right)$ for all $p=1,2, \ldots, k$,

Case 1. If,

$$
\begin{aligned}
\forall p & =1,2, \ldots, k, i_{p}<i_{p}^{\prime} \Longrightarrow\left(\frac{\sum_{p=1}^{k} i_{p}}{k}\right)<\left(\frac{\sum_{p=1}^{k} i_{p}^{\prime}}{k}\right) \\
& \Longrightarrow \operatorname{DH} 2 T A\left(\left(l_{i_{1}}, \varsigma_{i_{1}, j_{1}}\right),\left(l_{i_{2}}, \varsigma_{i_{2}, j_{2}}\right), \ldots,\left(l_{i_{k}}, \varsigma_{i_{k}, j_{k}}\right)\right) \\
& <\operatorname{DH} 2 T A\left(\left(l_{i_{1}}^{\prime}, \varsigma_{i_{1}, j_{1}}^{\prime}\right),\left(l_{i_{2}}^{\prime}, \varsigma_{i_{2}, j_{2}}^{\prime}\right), \ldots,\left(l_{i_{k}}^{\prime}, \varsigma_{i_{k}, j_{k}}^{\prime}\right)\right)
\end{aligned}
$$

Case 2. If $i_{p}=i_{p}^{\prime}$ which implies that $\left(\frac{\sum_{p=1}^{k} i_{p}}{k}\right)=\left(\frac{\sum_{p=1}^{k} i_{p}^{\prime}}{k}\right)=i$.

Let

$$
\begin{aligned}
& \operatorname{DH} 2 T A\left(\left(l_{i_{1}}, \varsigma_{i_{1}, j_{1}}\right),\left(l_{i_{2}}, \varsigma_{i_{2}, j_{2}}\right), \ldots,\left(l_{i_{k}}, \varsigma_{i_{k}, j_{k}}\right)\right)=\left(l_{i}, \varsigma_{i, j}\right), \\
& \operatorname{DH} 2 T A\left(\left(l_{i_{1}}^{\prime}, \varsigma_{i_{1}, j_{1}}^{\prime}\right),\left(l_{i_{2}}^{\prime}, \varsigma_{i_{2}, j_{2}}^{\prime}\right), \ldots,\left(l_{i_{k}}^{\prime}, \varsigma_{i_{k}, j_{k}}^{\prime}\right)\right)=\left(l_{i}^{\prime}, \varsigma_{i, j}^{\prime}\right), \\
& \min \left(\left(l_{i_{1}}, \varsigma_{i_{1}, j_{1}}\right),\left(l_{i_{2}}, \varsigma_{i_{2}, j_{2}}\right), \ldots,\left(l_{i_{k}}, \varsigma_{i_{k}, j_{k}}\right)\right)=\left(l_{i}, \varsigma_{i, j}^{\min }\right), \\
& \min \left(\left(l_{i_{1}}^{\prime}, \varsigma_{i_{1}, j_{1}}^{\prime}\right),\left(l_{i_{2}}^{\prime}, \varsigma_{i_{2}, j_{2}}^{\prime}\right), \ldots,\left(l_{i_{k}}^{\prime}, \varsigma_{i_{k}, j_{k}}^{\prime}\right)\right)=\left(l_{i}^{\prime}, \varsigma_{i, j}^{\prime \min }\right), \\
& \max \left(\left(l_{i_{1}}, \varsigma_{i_{1}, j_{1}}\right),\left(l_{i_{2}}, \varsigma_{i_{2}, j_{2}}\right), \ldots,\left(l_{i_{k}}, \varsigma_{i_{k}, j_{k}}\right)\right)=\left(l_{i}, \varsigma_{i, j}^{\max }\right) \text { and } \\
& \max \left(\left(l_{i_{1}}^{\prime}, \varsigma_{i_{1}, j_{1}}^{\prime}\right),\left(l_{i_{2}}^{\prime}, \varsigma_{i_{2}, j_{2}}^{\prime}\right), \ldots,\left(l_{i_{k}}^{\prime}, \varsigma_{i_{k}, j_{k}}^{\prime}\right)\right)=\left(l_{i}^{\prime}, \varsigma_{i, j}^{\prime \max }\right)
\end{aligned}
$$


therefore by theorem 4.2 ,

$$
\left(l_{i}, \varsigma_{i, j}^{\min }\right) \leq \operatorname{DH} 2 T A\left(\left(l_{i_{1}}, \varsigma_{i_{1}, j_{1}}\right),\left(l_{i_{2}}, \varsigma_{i_{2}, j_{2}}\right), \ldots,\left(l_{i_{k}}, \varsigma_{i_{k}, j_{k}}\right)\right) \leq\left(l_{i}, \varsigma_{i, j}^{\max }\right),
$$

and

$$
\left(l_{i}^{\prime}, \varsigma_{i, j}^{\prime \min }\right) \leq \operatorname{DH} 2 T A\left(\left(l_{i_{1}}^{\prime}, \varsigma_{i_{1}, j_{1}}^{\prime}\right),\left(l_{i_{2}}^{\prime}, \varsigma_{i_{2}, j_{2}}^{\prime}\right), \ldots,\left(l_{i_{k}}^{\prime}, \varsigma_{i_{k}, j_{k}}^{\prime}\right)\right) \leq\left(l_{i}^{\prime}, \varsigma_{i, j}^{\prime \max }\right) .
$$

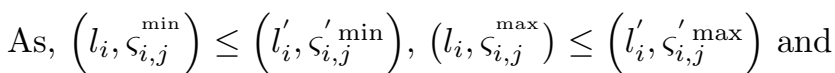

$\left(l_{i_{p}}, \varsigma_{i_{p}, j_{p}}\right) \leq\left(l_{i_{p}}^{\prime}, \varsigma_{i_{p}, j_{p}}^{\prime}\right)$, therefore $S\left(l_{i}, \varsigma_{i, j}^{\min }\right) \leq S\left(l_{i}^{\prime}, \varsigma_{i, j}^{\prime \min }\right), S\left(l_{i}, \varsigma_{i, j}^{\max }\right) \leq S\left(l_{i}^{\prime}, \varsigma_{i, j}^{\prime \max }\right)$ and $S\left(l_{i_{p}}, \varsigma_{i_{p}, j_{p}}\right) \leq S\left(l_{i_{p}}^{\prime}, \varsigma_{i_{p}, j_{p}}^{\prime}\right)$ for each $p$. Therefore by Theorem 4.2

$$
\begin{aligned}
& \operatorname{DH} 2 T A\left(\left(l_{i_{1}}, \varsigma_{i_{1}, j_{1}}\right),\left(l_{i_{2}}, \varsigma_{i_{2}, j_{2}}\right), \ldots,\left(l_{i_{k}}, \varsigma_{i_{k}, j_{k}}\right)\right) \\
\leq & \operatorname{DH} 2 T A\left(\left(l_{i_{1}}^{\prime}, \varsigma_{i_{1}, j_{1}}^{\prime}\right),\left(l_{i_{2}}^{\prime}, \varsigma_{i_{2}, j_{2}}^{\prime}\right), \ldots,\left(l_{i_{k}}^{\prime}, \varsigma_{i_{k}, j_{k}}^{\prime}\right)\right),
\end{aligned}
$$

which is required result.

Theorem 4.4. Let $\left(l_{i_{p}^{\prime}}, \varsigma_{i_{p}^{\prime}, j_{p}}\right)$ be a permutation of p hesitant 2-tuples linguistic elements of $\left(l_{i_{p}}, \varsigma_{i_{p}, j_{p}}\right)$, where $i_{p}, i_{p}^{\prime} \in\{0,1,2, \ldots, g\}, j_{p}, j_{p}^{\prime}=\{1,2, \ldots, n\}$ and $p=1,2, \ldots, k$ then,

$$
\begin{aligned}
& \operatorname{DH} 2 T A\left(\left(l_{i_{1}}, \varsigma_{i_{1}, j_{1}}\right),\left(l_{i_{2}}, \varsigma_{i_{2}, j_{2}}\right), \ldots,\left(l_{i_{k}}, \varsigma_{i_{k}, j_{k}}\right)\right) \\
= & \operatorname{DH} 2 T A\left(\left(l_{i_{1}}^{\prime}, \varsigma_{i_{1}, j_{1}}^{\prime}\right),\left(l_{i_{2}}^{\prime}, \varsigma_{i_{2}, j_{2}}^{\prime}\right), \ldots,\left(l_{i_{k}}^{\prime}, \varsigma_{i_{k}, j_{k}}^{\prime}\right)\right)
\end{aligned}
$$

Proof. Let us consider $(\sigma(1), \sigma(2), \ldots, \sigma(k))$ be permutation of $(1,2, \ldots, k)$ such that $\left(l_{i_{p}}, \varsigma_{i_{p}, j_{p}}\right)_{\sigma(1)} \leq\left(l_{i_{p}}, \varsigma_{i_{p}, j_{p}}\right)_{\sigma(2)} \leq \ldots \leq\left(l_{i_{p}}, \varsigma_{i_{p}, j_{p}}\right)_{\sigma(k)}$ then,

$$
\left(l_{i_{p}}, \varsigma_{i_{p}, j_{p}}\right)_{\sigma(p)}=\left(l_{i_{p}^{\prime}}, \varsigma_{i_{p}^{\prime}, j_{p}}\right)_{\sigma(p)} \forall p=1,2, \ldots, k
$$

therefore,

$$
\left(i_{p}\right)_{\sigma(p)}=\left(i_{p}^{\prime}\right)_{\sigma(p)} \Longrightarrow\left(\frac{\sum_{p=1}^{k} i_{p}}{k}\right)=\left(\frac{\sum_{p=1}^{k} i_{p}^{\prime}}{k}\right) \forall p=1,2, \ldots, k
$$

also,

$$
S\left(\left(l_{i_{p}}, \varsigma_{i_{p}, j_{p}}\right)_{\sigma(p)}\right)=S\left(\left(l_{i_{p}^{\prime}}, \varsigma_{i_{p}^{\prime}, j_{p}}\right)_{\sigma(p)}\right) \forall p=1,2, \ldots, k
$$

from equation 4.11 and 4.12

$$
\begin{aligned}
& \operatorname{DH} 2 T A\left(\left(l_{i_{1}}, \varsigma_{i_{1}, j_{1}}\right),\left(l_{i_{2}}, \varsigma_{i_{2}, j_{2}}\right), \ldots,\left(l_{i_{k}}, \varsigma_{i_{k}, j_{k}}\right)\right) \\
= & \operatorname{DH} 2 T A\left(\left(l_{i_{1}}^{\prime}, \varsigma_{i_{1}, j_{1}}^{\prime}\right),\left(l_{i_{2}}^{\prime}, \varsigma_{i_{2}, j_{2}}^{\prime}\right), \ldots,\left(l_{i_{k}}^{\prime}, \varsigma_{i_{k}, j_{k}}^{\prime}\right)\right) .
\end{aligned}
$$




\section{Hesitant 2-tuple Linguistic Information Aggregation Operators Based on the Choquet} INTEGRAL

In this section, we develop diminishing Choquet hesitant 2-tuple average operator (DCH2T A) by selecting Choquet integral to find the weights for $D W H 2 T A$. We also discussed different properties of $D C H 2 T A$.

Definition 5.1. Let $h_{1}=\left(l_{i_{1}}, \varsigma_{i_{1}, j_{1}}\right), h_{2}=\left(l_{i_{2}}, \varsigma_{i_{2}, j_{2}}\right), \ldots, h_{k}=\left(l_{i_{k}}, \varsigma_{i_{k}, j_{k}}\right)$ be $k$, 2-tuple hesitant linguistic terms where $j_{p}$ be the cardinality of $\varsigma_{i_{p}, j_{p}}$ for any finite natural number $p$. $X$ be the set of attributes and $\alpha$ be the fuzzy measure on $X$, then diminishing Choquet hesitant 2-tuple average operator (DCH2TA) is defined as follow:

$$
\begin{gathered}
\operatorname{DCH} 2 T A_{\alpha}\left(h_{1}, h_{2}, \ldots, h_{k}\right) \\
=\operatorname{DH} 2 T A\left(\left\{\left(\alpha\left(H_{\sigma(p)}\right)-\alpha\left(H_{\sigma(p-1)}\right)\right)\left(l_{i}, \varsigma_{i, j}\right)_{\sigma(p)} \mid p=1,2, \ldots, k\right\}\right),
\end{gathered}
$$

here $(\sigma(1), \sigma(2), \ldots, \sigma(k))$ be the permutation of $(1,2, \ldots, k)$ such that $\left(l_{i}, \varsigma_{i, j}\right)_{\sigma(1)} \geq\left(l_{i}, \varsigma_{i, j}\right)_{\sigma(2)} \geq \ldots \geq$ $\left(l_{i}, \varsigma_{i, j}\right)_{\sigma(k)}, X_{\sigma(p)}$ is the attribute corresponding to $\left(l_{i}, \varsigma_{i, j}\right)_{\sigma(p)}$ and $H_{\sigma(p)}=\left\{x_{\sigma(l)} \mid l \leq p\right\}$ for $p \geq 1, H_{\sigma(0)}=$ $\emptyset$.

Theorem 5.1. Let $\left(l_{i_{1}}, \varsigma_{i_{1}, j_{1}}\right)=\left(l_{i_{2}}, \varsigma_{i_{2}, j_{2}}\right)=\ldots=\left(l_{i_{k}}, \varsigma_{i_{k}, j_{k}}\right)$ be all equal $k$, hesitant 2-tuples linguistic elements such that $i_{1}=i_{2}=\ldots=i_{k}$ and $\varsigma_{i_{1}, j_{1}}=\varsigma_{i_{2}, j_{2}}=\ldots=\varsigma_{i_{k}, j_{k}}$. If $i_{p} \in\{0,1,2, \ldots, g\}, j_{p} \in\{1,2, \ldots, n\}$ and $p=1,2, \ldots, k, X$ is the set of attributes and $\alpha$ be the fuzzy measure on $X$, then diminishing Choquet hesitant 2-tuple average operator $(D C H 2 T A)$ is always

$$
\operatorname{DCH} 2 T A_{\alpha}\left(\left(l_{i_{1}}, \varsigma_{i_{1}, j_{1}}\right),\left(l_{i_{2}}, \varsigma_{i_{2}, j_{2}}\right), \ldots,\left(l_{i_{k}}, \varsigma_{i_{k}, j_{k}}\right)\right)=\left(l_{i}, \varsigma_{i, j}\right)
$$

where $\left(l_{i}, \varsigma_{i, j}\right)=w_{p}\left(l_{i_{p}}, \varsigma_{i_{p}, j_{p}}\right)_{p}, w_{p}=\alpha\left(H_{\sigma(p)}\right)-\alpha\left(H_{\sigma(p-1)}\right)=\frac{1}{k}$ and $(\sigma(1), \sigma(2), \ldots, \sigma(k))$ be the permutation of $(1,2, \ldots, k)$.

Proof. Given that,

$$
\begin{aligned}
\left(l_{i_{p}}, \varsigma_{i_{p}, j_{p}}\right)_{\sigma(p)} & =\left(l_{i_{p}}, \varsigma_{i_{p}, j_{p}}\right)_{\sigma(p-1)} \forall p=1,2,3, \ldots, k, \text { therefore } \\
\left(l_{i_{p}}\right)_{\sigma(p)} & =\left(l_{i_{p}}\right)_{\sigma(p-1)} \text { also }\left(\varsigma_{i_{p}, j_{p}}\right)_{\sigma(p)}=\left(\varsigma_{i_{p}, j_{p}}\right)_{\sigma(p-1)} \forall p=1,2,3, \ldots, k
\end{aligned}
$$

by definition 3.5 scalar product for $w_{p-1}$ and $w_{p}$ are

$$
\begin{aligned}
& A_{1}=\left\{\begin{array}{c}
(\mu)_{\sigma(p)} \mid(\mu)_{\sigma(p)}=(g+1)\left(1-\left(1-\frac{\left.g+\left(\gamma_{\left.i_{p}, j_{p}\right)_{\sigma(p)}}\right)^{w_{p}}\right)}{g+1}\right\}\right. \\
\mid \forall\left(\gamma_{i_{p}, j_{p}}\right)_{\sigma(p)} \in\left(\varsigma_{i_{p}, j_{p}}\right)_{\sigma(p)}
\end{array}\right. \\
& \text { and } B_{1}=\left\{\begin{array}{c}
(\mu)_{\sigma(p-1)} \mid(\mu)_{\sigma(p-1)}=(g+1)\left(1-\left(1-\frac{g+\left(\gamma_{\left.i_{p}, j_{p}\right)_{\sigma(p-1)}}\right)^{w_{p-1}}}{g+1}\right)\right. \\
\mid \forall\left(\gamma_{i_{p}, j_{p}}\right)_{\sigma(p-1)} \in\left(\varsigma_{i_{p}, j_{p}}\right)_{\sigma(p-1)}
\end{array}\right\}
\end{aligned}
$$


this implies that,

$$
A_{1}=B_{1} \text { as } w_{p-1}=w_{p} \text { and }\left(l_{i_{p}}, \varsigma_{i_{p}, j_{p}}\right)_{\sigma(p)}=\left(l_{i_{p}}, \varsigma_{i_{p}, j_{p}}\right)_{\sigma(p-1)} \forall p=1,2,3, \ldots, k \text {. }
$$

Let,

$$
\begin{aligned}
& A_{2}=\bigcup\left\{\theta_{1_{\sigma(p)}} \mid \theta_{1_{\sigma(p)}}=\operatorname{round}\left((\mu)_{\sigma(p)}\right), \forall(\mu)_{\sigma(p)} \in A_{1}\right\} \text { and } \\
& B_{2}=\bigcup\left\{\theta_{1_{\sigma(p-1)}} \mid \theta_{1_{\sigma(p-1)}}=\operatorname{round}\left((\mu)_{\sigma(p-1)}\right), \forall(\mu)_{\sigma(p-1)} \in B_{1}\right\} .
\end{aligned}
$$

By equation 5.2

$$
A_{2}=B_{2}
$$

As $\left(i_{p}\right)_{\sigma(p)}=\left(i_{p}\right)_{\sigma(p-1)}$ therefore by equation 5.3 ,

$$
\frac{\left(\sum_{\theta_{1}(p)} \in A_{2} \theta_{1_{\sigma(p)}}+\left(i_{p}\right)_{\sigma(p)}\right)}{\left(\left|A_{2}\right|+1\right)}=\frac{\left(\sum_{\theta_{1_{\sigma(p-1)} \in B_{2}}} \theta_{1_{\sigma(p-1)}}+\left(i_{p}\right)_{\sigma(p-1)}\right)}{\left(\left|B_{2}\right|+1\right)}=i
$$

and

$$
\begin{aligned}
\left(\varsigma_{i_{p}, j_{p}}\right)_{\sigma(p)} & =\left(\varsigma_{i_{p}, j_{p}}\right)_{\sigma(p-1)} \text { for all } p \\
& \Longrightarrow \bigcup_{p=1}^{k}\left(\varsigma_{i_{p}, j_{p}}\right)_{\sigma(p)}=\bigcup_{p=1}^{k}\left(\varsigma_{i_{p}, j_{p}}\right)_{\sigma(p-1)}=\eta_{1}(\text { say })
\end{aligned}
$$

where,

$$
\left(\varsigma_{i_{p}, j_{p}}\right)_{\sigma(p)}=\left\{\theta_{2_{\sigma(p)}} \mid \theta_{2_{\sigma(p)}}=\operatorname{different}\left((\mu)_{\sigma(p)}-\theta_{1_{\sigma(p)}}\right)\right\}
$$

and

$$
\left(\varsigma_{i_{p}, j_{p}}\right)_{\sigma(p-1)}=\left\{\theta_{2_{\sigma(p-1)}} \mid \theta_{2_{\sigma(p-1)}}=\operatorname{different}\left((\mu)_{\sigma(p-1)}-\theta_{1_{\sigma(p-1)}}\right)\right\} .
$$

As

$$
\begin{aligned}
\left(\varsigma_{i_{p}, j_{p}}\right)_{\sigma(p)} & =\left(\varsigma_{i_{p}, j_{p}}\right)_{\sigma(p-1)} \text { for all } p, \\
& \Longrightarrow \max \left(\varsigma_{i_{p}, j_{p}}\right)_{\sigma(p)}=\max \left(\varsigma_{i_{p}, j_{p}}\right)_{\sigma(p-1)} \text { for all } p
\end{aligned}
$$

therefore

$$
\min \left(\bigcup_{p=1}^{k}\left\{\max \left(\varsigma_{i_{p}, j_{p}}\right)_{\sigma(p)}\right\}\right)=\min \left(\bigcup_{p=1}^{k}\left\{\max \left(\varsigma_{i_{p}, j_{p}}\right)_{\sigma(p-1)}\right\}\right)
$$

and

$$
\max \left(\bigcup_{p=1}^{k}\left\{\min \left(\varsigma_{i_{p}, j_{p}}\right)_{\sigma(p)}\right\}\right)=\max \left(\bigcup_{p=1}^{k}\left\{\min \left(\varsigma_{i_{p}, j_{p}}\right)_{\sigma(p-1)}\right\}\right)
$$


By equation 5.4 let $r_{q} \in \eta_{1}$ then,

$$
\begin{aligned}
& \left(\varsigma_{1}\right)_{\sigma(p)}=\bigcup_{q=1}^{\left|\eta_{1}\right|}\left\{\min \left(r_{q}, \max \left(\begin{array}{c}
\min \left(\bigcup_{p=1}^{k}\left\{\max \left(\varsigma_{i_{p}, j_{p}}\right)_{\sigma(p)}\right\}\right), \\
\max \left(\bigcup_{p=1}^{k}\left\{\min \left(\varsigma_{i_{p}, j_{p}}\right)_{\sigma(p)}\right\}\right.
\end{array}\right)\right)\right\}
\end{aligned}
$$

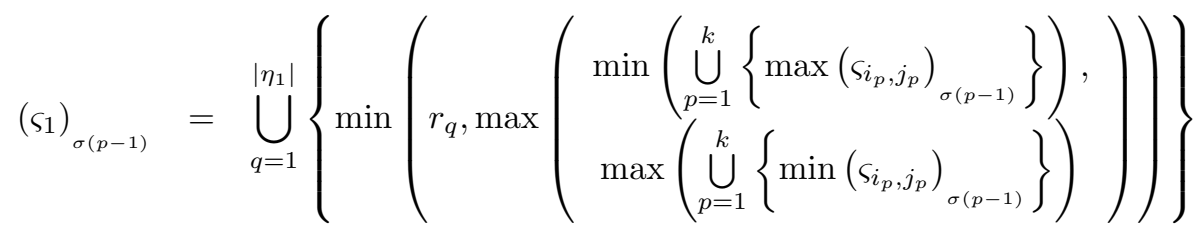

and

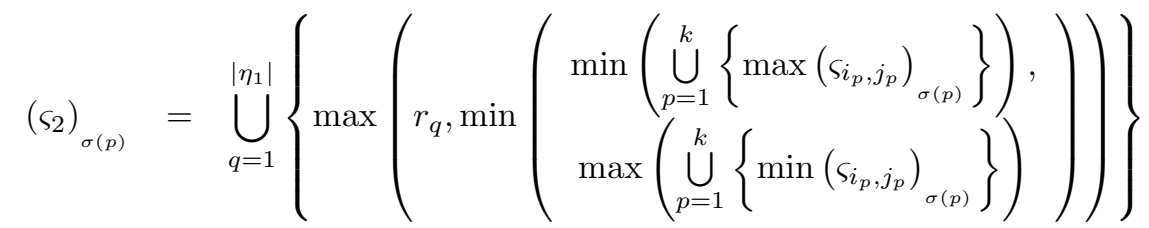

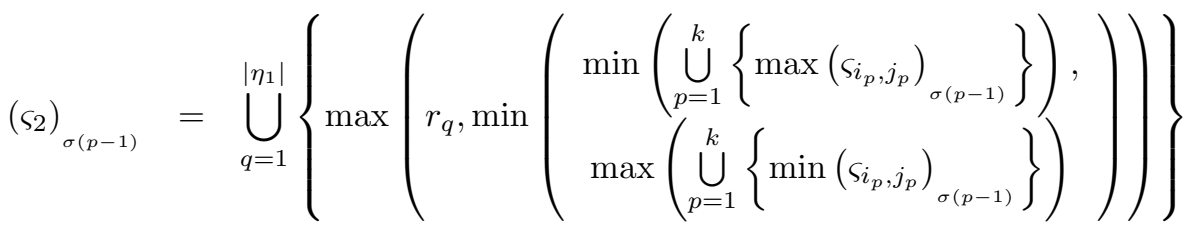

by equations 5.2,5.3 and 5.4 we have, $\left(\varsigma_{1}\right)_{\sigma(p)}=\left(\varsigma_{1}\right)_{\sigma(p-1)}$ and $\left(\varsigma_{2}\right)_{\sigma(p)}=\left(\varsigma_{2}\right)_{\sigma(p-1)}$ therefore,

$$
\left(\varsigma_{1}\right)_{\sigma(p)} \cap\left(\varsigma_{2}\right)_{\sigma(p)}=\left(\varsigma_{1}\right)_{\sigma(p-1)} \cap\left(\varsigma_{2}\right)_{\sigma(p-1)}=\varsigma_{i, j} .
$$

Given that $w_{p}=\alpha\left(H_{\sigma(p)}\right)-\alpha\left(H_{\sigma(p-1)}\right)=\frac{1}{k}$ for all $p$, therefore by definition 3.5 and equation 5.5 we have,

$$
w_{p}\left(l_{i_{p}}, \varsigma_{i_{p}, j_{p}}\right)_{p}=w_{p-1}\left(l_{i_{p}}, \varsigma_{i_{p}, j_{p}}\right)_{p-1}=\left(l_{i}, \varsigma_{i, j}\right) \text { (say) }
$$

Therefore by theorem 4.1 we have required result,

$$
D C H 2 T A_{\alpha}\left(\left(l_{i_{1}}, \varsigma_{i_{1}, j_{1}}\right),\left(l_{i_{2}}, \varsigma_{i_{2}, j_{2}}\right), \ldots,\left(l_{i_{k}}, \varsigma_{i_{k}, j_{k}}\right)\right)=\left(l_{i}, \varsigma_{i, j}\right)
$$

Theorem 5.2. Let $\left(l_{i_{p}}, \varsigma_{i_{p}, j_{p}}\right)$ be $k$, hesitant 2-tuples linguistic elements, if $i_{p} \in\{0,1,2, \ldots, g\}, j_{p} \in$ $\{1,2, \ldots, n\}$ and $p=1,2, \ldots, k . X$ be the set of attributes and $\alpha$ be the fuzzy measure on $X$, then for any $g$ use as upper limit of the linguistic term set, then diminishing Choquet hesitant 2-tuple average operator (DCH2TA) must satisfied,

$$
\left(l_{i_{p}}, \min \left(\varsigma_{i_{p}, j_{p}}\right)\right)_{\sigma(k)} \leq \operatorname{DCH} 2 T A_{\alpha}\left(h_{1}, h_{2}, \ldots, h_{k}\right) \leq\left(l_{i_{p}}, \max \left(\varsigma_{i_{p}, j_{p}}\right)\right)_{\sigma(1)}
$$

where $(\sigma(1), \sigma(2), \ldots, \sigma(k))$ be the permutation of $(1,2, \ldots, k)$ such that $\left(l_{i_{p}}, \varsigma_{i_{p}, j_{p}}\right)_{\sigma(k)} \leq\left(l_{i_{p}}, \varsigma_{i_{p}, j_{p}}\right)_{\sigma(k-1)} \leq$ $\ldots \leq\left(l_{i_{p}}, \varsigma_{i_{p}, j_{p}}\right)_{\sigma(1)}$. 
Proof. As,

$$
\left(l_{i_{p}}, \varsigma_{i_{p}, j_{p}}\right)_{\sigma(k)} \leq\left(l_{i_{p}}, \varsigma_{i_{p}, j_{p}}\right)_{\sigma(k-1)} \leq \ldots \leq\left(l_{i_{p}}, \varsigma_{\left.i_{p}, j_{p}\right)_{\sigma(1)}}\right.
$$

also

$$
\left(l_{i_{p}}, \min \left(\varsigma_{i_{p}, j_{p}}\right)\right)_{\sigma(k)} \leq\left(l_{i_{p}}, \varsigma_{i_{p}, j_{p}}\right)_{\sigma(k)} \text { and }\left(l_{i_{p}}, \varsigma_{i_{p}, j_{p}}\right)_{\sigma(1)} \leq\left(l_{i_{p}}, \max \left(\varsigma_{i_{p}, j_{p}}\right)\right)_{\sigma(1)} \text {. }
$$

Therefore

$$
\begin{aligned}
\left(l_{i_{p}}, \min \left(\varsigma_{i_{p}, j_{p}}\right)\right)_{\sigma(k)} & \leq\left(l_{i_{p}}, \varsigma_{i_{p}, j_{p}}\right)_{\sigma(k)} \leq\left(l_{i_{p}}, \varsigma_{i_{p}, j_{p}}\right)_{\sigma(k-1)} \\
& \leq \ldots \leq\left(l_{i_{p}}, \varsigma_{\left.i_{p}, j_{p}\right)_{\sigma(1)}} \leq\left(l_{i_{p}}, \max \left(\varsigma_{i_{p}, j_{p}}\right)\right)_{\sigma(1)} .\right.
\end{aligned}
$$

Because,

$$
0 \leq \alpha\left(H_{\sigma(p)}\right)-\alpha\left(H_{\sigma(p-1)}\right) \leq 1 \forall p=1,2,3, \ldots, k
$$

Therefore by definition 3.5 we have,

$$
\begin{aligned}
& \text { Let } \tau_{p}=\alpha\left(H_{\sigma(p)}\right)-\alpha\left(H_{\sigma(p-1)}\right)\left(l_{i_{p}}, \varsigma_{i_{p}}, j_{p}\right) \forall p=1,2, \ldots, k \\
& \tau_{p}=\left\{\begin{array}{c}
\mu_{2} \mid \mu_{2}=(g+1)\left(1-\left(1-\frac{g+\left(\gamma_{i_{p}, j_{p}}\right)}{g+1}\right)^{\alpha\left(H_{\sigma(p)}\right)-\alpha\left(H_{\sigma(p-1)}\right)}\right) \\
\forall\left(\gamma_{i_{p}, j_{p}}\right) \in\left(\varsigma_{i_{p}, j_{p}}\right) \text { where } p=1,2, \ldots k
\end{array}\right\} \\
& \text { and } \beta_{p}=\bigcup\left\{\theta_{2} \mid \theta_{2}=\operatorname{round}\left(\mu_{2}\right), \forall \mu_{2} \in \tau_{p}\right\} \\
& \Longrightarrow \lambda_{p}=\frac{\left(\sum_{\theta_{2} \in \beta_{p}} \theta_{2}+i_{p}\right)}{\left|\beta_{p}\right|+1},\left|\beta_{p}\right| \text { be cardinality of } \beta_{p} \text {. }
\end{aligned}
$$

Clearly,

$$
\left(i_{p}\right)_{\sigma(k)} \leq \operatorname{round}\left(\frac{\sum \lambda_{p}}{k}\right)=i^{\prime} \leq\left(i_{p}\right)_{\sigma(1)} .
$$

Let $l_{i^{\prime}}$ be the linguistic term of $D C H 2 T A_{\alpha}\left(\left(l_{i_{1}}, \varsigma_{i_{1}, j_{1}}\right),\left(l_{i_{2}}, \varsigma_{i_{2}, j_{2}}\right), \ldots,\left(l_{i_{k}}, \varsigma_{i_{k}, j_{k}}\right)\right)$. By definition 5.1 we observe that translation of $l_{i^{\prime}}$, (say $\left.\varsigma_{i^{\prime}, j^{\prime}}\right)$ may truncate the extreme values of $\left(\varsigma_{i_{p}, j_{p}}\right)_{\sigma(k)}$ and $\left(\varsigma_{i_{p}, j_{p}}\right)_{\sigma(1)}$ i.e. it must satisfied the following condition,

$$
\min \left(\left(\varsigma_{i_{p}, j_{p}}\right)_{\sigma(k)}\right) \leq \gamma_{i_{p}, j_{p}}^{\prime} \leq \max \left(\left(\varsigma_{i_{p}, j_{p}}\right)_{\sigma(1)}\right) \forall \gamma_{i_{p}, j_{p}}^{\prime} \in \varsigma_{i^{\prime}, j^{\prime}}
$$

therefore, from equation 5.6 and 5.7 we have,

$$
\begin{gathered}
\left(l_{i_{p}}, \min \left(\varsigma_{i_{p}, j_{p}}\right)\right)_{\sigma(k)} \leq \\
\quad D C H 2 T A_{\alpha}\left(\left(l_{i_{1}}, \varsigma_{i_{1}, j_{1}}\right),\left(l_{i_{2}}, \varsigma_{i_{2}, j_{2}}\right), \ldots,\left(l_{i_{k}}, \varsigma_{i_{k}, j_{k}}\right)\right) \\
\leq\left(l_{i_{p}}, \max \left(\varsigma_{i_{p}, j_{p}}\right)\right)_{\sigma(1)}
\end{gathered}
$$


Theorem 5.3. Let $\left(l_{i_{p}}, \varsigma_{i_{p}, j_{p}}\right) \leq\left(l_{i_{p}^{\prime}}^{\prime}, \varsigma_{i_{p}^{\prime}, j_{p}^{\prime}}^{\prime}\right)$ for all $p=1,2, \ldots, k$ if for $i_{p}, i_{p}^{\prime} \in\{0,1,2, \ldots, g\}, j_{p}, j_{p}^{\prime} \in$ $\{1,2, \ldots, n\}$. Let $X$ be the set of attributes and $\alpha$ be the fuzzy measure on $X$, then,

$$
\begin{array}{r}
\operatorname{DCH} 2 T A_{\alpha}\left(\left(l_{i_{1}}, \varsigma_{i_{1}, j_{1}}\right),\left(l_{i_{2}}, \varsigma_{i_{2}, j_{2}}\right), \ldots,\left(l_{i_{k}}, \varsigma_{i_{k}, j_{k}}\right)\right) \\
\leq \quad \operatorname{DCH} 2 T A_{\alpha}\left(\left(l_{i_{1}^{\prime}}^{\prime}, \varsigma_{i_{1}^{\prime}, j_{1}^{\prime}}^{\prime}\right),\left(l_{i_{2}}^{\prime}, \varsigma_{i_{2}, j_{2}^{\prime}}^{\prime}\right), \ldots,\left(l_{i_{k}^{\prime}}^{\prime}, \varsigma_{i_{k}, j_{k}^{\prime}}^{\prime}\right)\right)
\end{array}
$$

Proof. If $l_{i_{p}}=l_{i_{p}^{\prime}}^{\prime}$ then order of $\left(l_{i_{p}}, \varsigma_{i_{p}, j_{p}}\right)$ and $\left(l_{i_{p}^{\prime}}^{\prime}, \varsigma_{i_{p}^{\prime}, j_{p}^{\prime}}^{\prime}\right)$ depend on possible translations of $l_{i_{p}}$ and $l_{i_{p}^{\prime}}^{\prime}$. As, $\left(l_{i_{p}}, \varsigma_{i_{p}, j_{p}}\right)_{\sigma(p)} \leq\left(l_{i_{p}^{\prime}}^{\prime}, \varsigma_{i_{p}^{\prime}, j_{p}^{\prime}}^{\prime}\right)_{\sigma(p)} \forall p=1,2,3, \ldots, k$. Therefore,

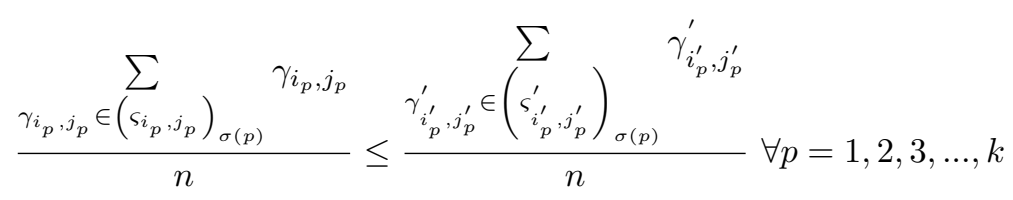

We also know that

$$
\begin{aligned}
& 0 \leq\left(\alpha\left(H_{\sigma(p)}\right)-\alpha\left(H_{\sigma(p-1)}\right)\right) \leq 1 \forall p=1,2,3, \ldots, k \text { also } \\
& 0 \leq\left(\alpha\left(H_{\sigma(p)}^{\prime}\right)-\alpha\left(H_{\sigma(p-1)}^{\prime}\right)\right) \leq 1 \forall p=1,2,3, \ldots, k
\end{aligned}
$$

Implies that

$$
\begin{aligned}
& \beta_{1}=\alpha\left(H_{\sigma(p)}\right)-\alpha\left(H_{\sigma(p-1)}\right)\left(l_{i_{p}}, \varsigma_{i_{p}, j_{p}}\right)_{\sigma(p)} \\
& =\left\{\begin{array}{c}
\mu \mid \mu=(g+1)\left(1-\left(1-\frac{g+\left(\gamma_{i_{p}, j_{p}}\right)_{\sigma(p)}}{g+1}\right)^{\alpha\left(H_{\sigma(p)}\right)-\alpha\left(H_{\sigma(p-1)}\right)}\right) \\
\forall\left(\gamma_{i_{p}, j_{p}}\right)_{\sigma(p)} \in\left(\varsigma_{i_{p}, j_{p}}\right)_{\sigma(p)}
\end{array}\right\}
\end{aligned}
$$

Let

$$
\begin{aligned}
& \beta_{2}=\bigcup\left\{\theta_{1} \mid \theta_{1}=\operatorname{round}(\mu), \forall \mu \in \beta_{1}\right\} \\
& \Longrightarrow(i)_{\sigma(p)}=\frac{\left(\sum_{\theta_{1} \in \beta_{2}} \theta_{1}+\left(i_{p}\right)_{\sigma(p)}\right)}{\left|\beta_{2}\right|+1},\left|\beta_{2}\right| \text { be cardinality of } \beta_{2} \\
& \beta_{1}^{\prime}=\alpha\left(H_{\sigma(p)}\right)-\alpha\left(H_{\sigma(p-1)}\right)\left(l_{i_{p}}, \varsigma_{i_{p}, j_{p}}\right) \\
& =\left\{\begin{array}{c}
\mu^{\prime} \mid \mu^{\prime}=(g+1)\left(1-\left(1-\frac{g+\left(\gamma_{i_{p}^{\prime}, j_{p}^{\prime}}^{\prime}\right)_{\sigma(p)}}{g+1}\right)^{\alpha\left(H_{\sigma(p)}^{\prime}\right)-\alpha\left(H_{\sigma(p-1)}^{\prime}\right)}\right. \\
\forall\left(\gamma_{i_{p}^{\prime}, j_{p}^{\prime}}^{\prime}\right)_{\sigma(p)} \in\left(\varsigma_{i_{p}^{\prime}, j_{p}^{\prime}}^{\prime}\right)_{\sigma(p)}
\end{array}\right\}
\end{aligned}
$$


Let

$$
\begin{aligned}
\beta_{2}^{\prime} & =\bigcup\left\{\theta_{1}^{\prime} \mid \theta_{1}^{\prime}=\operatorname{round}\left(\mu^{\prime}\right), \forall \mu^{\prime} \in \beta_{1}^{\prime}\right\} \\
\Longrightarrow & \left(i^{\prime}\right)_{\sigma(p)}=\frac{\left(\sum_{\theta_{1}^{\prime} \in \beta_{2}^{\prime}} \theta_{1}^{\prime}+\left(i_{p}^{\prime}\right)_{\sigma(p)}\right)}{\left|\beta_{2}^{\prime}\right|+1},\left|\beta_{2}^{\prime}\right| \text { be cardinality of } \beta_{2}^{\prime}
\end{aligned}
$$

From equations 5.8, 5.9 and 5.10 we have,

$$
D C H 2 T A_{\alpha}\left(h_{1}, h_{2}, \ldots, h_{k}\right) \leq D C H 2 T A_{\alpha}\left(h_{1}^{\prime}, h_{2}^{\prime}, \ldots, h_{k}^{\prime}\right)
$$

If $i_{p}<i_{p}^{\prime}$ then obviously from equation 5.9 and 5.10 we have

$$
(i)_{\sigma(p)} \leq\left(i^{\prime}\right)_{\sigma(p)} \Longrightarrow \operatorname{DCH} 2 T A_{\alpha}\left(h_{1}, h_{2}, \ldots, h_{k}\right) \leq \operatorname{DCH} 2 T A_{\alpha}\left(h_{1}^{\prime}, h_{2}^{\prime}, \ldots, h_{k}^{\prime}\right)
$$

Theorem 5.4. Let $h_{p}^{\prime}=\left(l_{i_{p}^{\prime}}^{\prime}, \varsigma_{i_{p}^{\prime}, j_{p}}^{\prime}\right)_{p}$ be a permutation of $p$ hesitant 2-tuples linguistic elements of $h_{p}=$ $\left(l_{i_{p}}, \varsigma_{i_{p}, j_{p}}\right)_{p}$. Where $i_{p}=0,1,2, \ldots, g, j_{p}=1,2, \ldots, n$ and $p=1,2, \ldots, k . X$ be the set of attributes and $\alpha$ be the fuzzy measure on $X$, then,

$$
D C H 2 T A_{\alpha}\left(h_{1}, h_{2}, \ldots, h_{k}\right)=D C H 2 T A_{\alpha}\left(h_{1}^{\prime}, h_{2}^{\prime}, \ldots, h_{k}^{\prime}\right)
$$

Proof. Let us consider $(\sigma(1), \sigma(2), \ldots, \sigma(k))$ be permutation of $(1,2, \ldots, k)$ such that $\left(l_{i_{p}}, \varsigma_{i_{p}, j_{p}}\right)_{\sigma(1)} \leq\left(l_{i_{p}}, \varsigma_{i_{p}, j_{p}}\right)_{\sigma(2)} \leq \ldots \leq\left(l_{i_{p}}, \varsigma_{i_{p}, j_{p}}\right)_{\sigma(k)}$ then

$$
\left(l_{i_{p}}, \varsigma_{i_{p}, j_{p}}\right)_{\sigma(p)}=\left(l_{i_{p}^{\prime}}^{\prime}, \varsigma_{i_{p}^{\prime}, j_{p}^{\prime}}^{\prime}\right)_{\sigma(p)} \text { such that } i_{p}=i_{p}^{\prime} \text { and } \varsigma_{i_{p}, j_{p}}=\varsigma_{i_{p}^{\prime}, j_{p}^{\prime}}^{\prime} \forall p=1,2,3, \ldots, k
$$

We also know that

$$
0 \leq\left(\alpha\left(H_{\sigma(p)}\right)-\alpha\left(H_{\sigma(p-1)}\right)\right) \leq 1 \forall p=1,2,3, \ldots, k
$$

Implies that

$$
\begin{aligned}
\beta_{1}= & \alpha\left(H_{\sigma(p)}\right)-\alpha\left(H_{\sigma(p-1)}\right)\left(l_{i_{p}}, \varsigma_{i_{p}, j_{p}}\right)_{\sigma(k)} \\
= & \left\{\begin{array}{c}
\mu \mid \mu=(g+1)\left(1-\left(1-\frac{g+\left(\gamma_{i_{p}, j_{p}}\right)_{\sigma(p)}}{g+1}\right)^{\alpha\left(H_{\sigma(p)}\right)-\alpha\left(H_{\sigma(p-1)}\right)}\right. \\
\forall\left(\gamma_{i_{p}, j_{p}}\right)_{\sigma(p)} \in\left(\varsigma_{i_{p}, j_{p}}\right)_{\sigma(p)}
\end{array}\right\} .
\end{aligned}
$$

Let $\beta_{2}=\bigcup\left\{\theta_{1} \mid \theta_{1}=\operatorname{round}(\mu), \forall \mu \in \beta_{1}\right\}$

$$
\Longrightarrow(i)_{\sigma(p)}=\frac{\left(\sum_{\theta_{1} \in \beta_{2}} \theta_{1}+\left(i_{p}\right)_{\sigma(k)}\right)}{\left|\beta_{2}\right|+1},\left|\beta_{2}\right| \text { be cardinality of } \beta_{2}
$$




$$
\begin{aligned}
\beta_{1}^{\prime} & =\alpha\left(H_{\sigma(p)}\right)-\alpha\left(H_{\sigma(p-1)}\right)\left(l_{i_{p}}, \varsigma_{i_{p}, j_{p}}\right) \\
& =\left\{\begin{array}{c}
\mu^{\prime} \mid \mu^{\prime}=(g+1)\left(1-\left(1-\frac{g+\left(\gamma_{i_{p}^{\prime}, j_{p}^{\prime}}^{\prime}\right)_{\sigma(p)}}{g+1}\right)^{\alpha\left(H_{\sigma(p)}^{\prime}\right)-\alpha\left(H_{\sigma(p-1)}^{\prime}\right)}\right. \\
\forall\left(\gamma_{i_{p}^{\prime}, j_{p}^{\prime}}^{\prime}\right)_{\sigma(p)} \in\left(\varsigma_{i_{p}^{\prime}, j_{p}^{\prime}}^{\prime}\right)_{\sigma(p)}
\end{array}\right\} . \\
\text { Let } \beta_{2}^{\prime}= & \bigcup\left\{\theta_{1}^{\prime} \mid \theta_{1}^{\prime}=\operatorname{round}\left(\mu^{\prime}\right), \forall \mu^{\prime} \in \beta_{1}^{\prime}\right\} \\
& \Longrightarrow\left(i^{\prime}\right)_{\sigma(p)}=\frac{\left(\sum_{\theta_{1}^{\prime} \in \beta_{2}^{\prime}} \theta_{1}^{\prime}+\left(i_{p}^{\prime}\right)_{\sigma(p)}\right)}{\left|\beta_{2}^{\prime}\right|+1},\left|\beta_{2}^{\prime}\right| \text { be cardinality of } \beta_{2}^{\prime} .
\end{aligned}
$$

From equations 5.11, 5.12 and 5.13

$$
\begin{gathered}
\beta_{1}=\beta_{1}^{\prime} \text { and } \beta_{2}=\beta_{2}^{\prime} \forall p=1,2,3, \ldots, k \\
\Longrightarrow D C H 2 T A_{\alpha}\left(h_{1}, h_{2}, \ldots, h_{k}\right)=C H 2 T N_{\alpha}\left(h_{1}^{\prime}, h_{2}^{\prime}, \ldots, h_{k}^{\prime}\right)
\end{gathered}
$$

which is required proof.

\section{An Application of DCH2TA Operators to Multiple Attribute Decision Making}

In this section $D C H 2 T A$ operator is applied to multiple attribute decision making problems based on hesitant 2-tuple linguistic information. Firstly, we developed a decision making method for utilization of $D C H 2 T A$ operator.

Let $D=\left\{D_{1}, D_{2}, \ldots, D_{r}\right\}$ be the set of " $r$ " decision makers, $X=\left\{x_{1}, x_{2}, \ldots, x_{m}\right\}$ be the set of alternatives and $Y=\left\{y_{1}, y_{2}, \ldots, y_{n}\right\}$ be the set of attributes.

Step 1. The decision makers developed the decision matrices $M_{p}=\left[\left(l_{i j k}^{p}, \varsigma^{p}\right)\right]_{m \times n}$, where $\left(l_{i j k}^{p}, \varsigma^{p}\right)$ be the hesitant evaluation of the alternatives $x_{i}$ determined by the decision makers $D_{p}$ based on attributes $y_{j}$, where $i=1,2, \ldots, m, j=1,2, \ldots, n$, and $p=1,2, . ., r$, where $\varsigma^{p} \subset[-0.5,0.5)$ and $k \in\{0,1,2, \ldots g\}$.

Step 2. Find the matrix Magg $=\left[\operatorname{DH} 2 T A\left(l_{i j k}^{p}, \varsigma^{p}\right)\right]_{m \times n}$, where, $D H 2 T A\left(l_{i j k}^{p}, \varsigma^{p}\right)$ is an aggregate value of $\left(l_{i j k}^{p}, \varsigma^{p}\right)(i=1,2, \ldots, m, j=1,2, \ldots, n)$ for all decision maker's evaluation as follow:

$$
\begin{aligned}
& \operatorname{DH} 2 \mathrm{TA}\left(\left(l_{i j k_{1}}^{1}, \varsigma^{1}\right),\left(l_{i j k_{2}}^{2}, \varsigma^{2}\right), \ldots,\left(l_{i j k_{n}}^{n}, \varsigma^{n}\right)\right) \\
= & \left(l_{k}, \varsigma_{k}\right) \text { where } k=\text { round }\left(\frac{k_{1}+k_{2}+\ldots+k_{n}}{n}\right) \text { and } \\
\varsigma_{k}= & \left\{x \mid x \in \varsigma_{1} \cap \varsigma_{2}\right\} \text { for all } r_{q} \in \eta=\bigcup_{j=1}^{n} \varsigma^{j} \text { we have }
\end{aligned}
$$




$$
\varsigma_{1}=\bigcup_{q=1}^{|\eta|}\left\{\min \left(r_{q}, \max \left(\begin{array}{c}
\min \left(\bigcup_{j=1}^{n}\left\{\max \left(\varsigma^{p}\right)\right\}\right), \\
\max \left(\bigcup_{j=1}^{n} \min \left\{\left(\varsigma^{p}\right)\right\}\right)
\end{array}\right)\right\}\right.
$$

and

$$
\varsigma_{2}=\bigcup_{q=1}^{|\eta|}\left\{\max \left(r_{q}, \min \left(\begin{array}{c}
\min \left(\bigcup_{j=1}^{n}\left\{\max \left(\varsigma^{p}\right)\right\}\right), \\
\max \left(\bigcup_{j=1}^{j}\left\{\min \left(\varsigma^{p}\right)\right\}\right)
\end{array}\right)\right)\right\}
$$

where round (*) be the round function and $|\eta|$ be the cardinality of $\eta$.

Step 3. Confirm the fuzzy measures of attributes sets of $B$. We use the $D C H 2 T A$ operator define in definition 5.1 to aggregate the values to find overall values $(l, \varsigma)_{i}(i=1,2, \ldots, m)$ of alternatives $A_{i}$.

$$
\begin{aligned}
(l, \varsigma)_{i} & =\operatorname{DCH} 2 T A_{\alpha}\left(\left(l_{i 1}, \varsigma_{i 1}\right),\left(l_{i 2}, \varsigma_{i 2}\right), \ldots,\left(l_{i n}, \varsigma_{i n}\right)\right) \\
& =\operatorname{DH} 2 T A\left(w_{i 1}\left(l_{i \sigma(1)}, \varsigma_{i \sigma(1)}\right), w_{i 2}\left(l_{i \sigma(2)}, \varsigma_{i \sigma(2)}\right), \ldots, w_{i n}\left(l_{i \sigma(n)}, \varsigma_{i \sigma(n)}\right)\right)
\end{aligned}
$$

where $\left(\sigma_{(1)}, \sigma_{(2)}, \ldots, \sigma_{(n)}\right)$ be the permutation of $(1,2, \ldots, n)$ such that

$$
\left(l_{i \sigma(1)}, \varsigma_{i \sigma(1)}\right) \geq\left(l_{i \sigma(2)}, \varsigma_{i \sigma(2)}\right) \geq \ldots \geq\left(l_{i \sigma(n)}, \varsigma_{i \sigma(n)}\right)
$$

and $w_{i j}=\alpha\left(H_{i \sigma(j)}\right)-\alpha\left(H_{i \sigma(j-1)}\right)$ is the set of attributes corresponding to $\left(l_{i \sigma(1)}\right.$, $\left.\varsigma_{i \sigma(1)}\right),\left(l_{i \sigma(2)}, \varsigma_{i \sigma(2)}\right), \ldots,\left(l_{i \sigma(n)}, \varsigma_{i \sigma(n)}\right)$.

Step 4. Rank these aggregate values $(l, \varsigma)_{i}(i=1,2, \ldots, m)$ in descending order according to the rule in definition 3.2 and select $(l, \varsigma)_{i}$ with the largest value.

\section{Illustrative EXAMPLE}

In order to demonstrate the significance of our newly proposed method, we consider an example where Mr. Robert, a food chain owner, wants to hire a supplier for raw food material for his chain. To save hedge risks, a three member committee (decision makers), $D=\left\{D_{1}, D_{2}, D_{3}\right\}$ has been created to select the most suitable supplier. Decision makers short listed five potential suppliers after initial analysis for supplier's capabilities. Let $S=\left\{s_{1}, s_{2}, s_{3}, s_{4}, s_{5}\right\}$ be the set of short listed suppliers. During the supplier selection process, decision maker decide to consider the following set of attributes for judgments $Y=\left\{Y_{1}\right.$ (price), $Y_{2}$ (quality), $Y_{3}$ (delivery time), $Y_{4}$ (financial status of the company)\}.

In numerous practical group decision making problems in supply chain management, the contractor selection or determination of an accomplice for an endeavor in the field of production network administration, military framework effectiveness assessment, etc. decision makers normally need to give their preferences over alternatives. As preference information given by decision makers is normally imprecise. It might be due 
to hesitations, uncertainty or vagueness about preferences as a decision should be made under time pressure and lack of information or knowledge processing capacities especially when financial condition turns out to be more complex. The best choice for decision maker is to handle data in hesitant 2-tuple elements due to effectiveness of them in these particular situations.

Consider that decision makers evaluate the alternatives with respect to the attributes in 2-tuple linguistic arguments to form decision matrices $M_{p}$ where $p=\{1,2,3\}$.

Step 1. Develop decision matrices $M_{p}=\left[\left(l_{i j k}^{p}, \varsigma^{p}\right)\right]_{5 \times 4}, \varsigma^{p} \subset[-0.5,0.5)$

$$
\begin{aligned}
& M_{1} \\
& =\left[\begin{array}{cccc}
(M,\{-0.3,0.0,0.2\}) & (G,\{0.45,0.32,0.2\}) & (P,\{0.2,0.3\}) & (P,\{-0.3,0.1\}) \\
(P,\{0.0,0.2,0.1\}) & (M,\{-0.48,-0.2,0.0\}) & (M,\{-0.45,0.1\}) & (G,\{-0.2,0.1,0.2\}) \\
(G,\{-0.3,0.1,0.2\}) & (M,\{-0.0,0.2\}) & (V G,\{-0.2 .0 .0,0.4\}) & (P,\{-0.3,0.1,0.2\}) \\
(V G,\{-0.1,0.0,0.2\}) & (P,\{0.0,0.2,0.4\}) & (P,\{-0.5,-0.3\}) & (M,\{-0.45,-0.25\}) \\
(E G,\{-0.4,-0.3,0.1\}) & (P,\{-0.1,0.2,0.3\}) & (V P,\{-0.45,-0.2\}) & (G,\{-0.4,-0.1,0.0\})
\end{array}\right] \\
& M_{2}=\left[\begin{array}{cccc}
(P,\{-0.3,-0.1\}) & (V G,\{-0.1,0.0,0.1\}) & (V P,\{-0.2,0.3\}) & (M,\{0.1,0.2,0.4\}) \\
(V P,\{0.4\}) & (P,\{0.2,0.3\}) & (G,\{0.3,0.4\}) & (V G,\{-0.1,-0.45,-0.2\}) \\
(M,\{0.0,0.3\}) & (P,\{-0.1,0.2\}) & (G,\{0.1,0.3\}) & (V P,\{-0.3,-0.2,0.0\}) \\
(E G,\{0.2,0.4\}) & (M,\{-0.4,0.3\}) & (P,\{0.2,0.4\}) & (G,\{0.1,0.3,0.4\}) \\
(G,\{-0.2,0.1\}) & (M,\{-0.2,0.15\}) & (P,\{-0.1,0.2\}) & (V G,\{-0.1,0.3\})
\end{array}\right] \\
& M_{3}=\left[\begin{array}{cccc}
(G,\{-0.5,0.1,0.2\}) & (V G,\{0.2,0.3\}) & (M,\{0.1,0.2\}) & (V P,\{0.0,0.1,0.2\}) \\
(M,\{-0.4,-0.1\}) & (P,\{0.0,0.2,0.4\}) & (V G,\{-0.3,-0.2\}) & (M,\{-0.2,-0.1,0.0\}) \\
(P,\{-0.2,0.0,0.1\}) & (V G,\{-0.05,0.2\}) & (G,\{0.0,0.1,0.25\}) & (V P,\{-0.3,-0.2,0.0\}) \\
(G,\{-0.3,-0.1,0.0\}) & (G,\{0.0,0.25,0.45\}) & (P,\{0.1,0.2,0.3\}) & (M,\{-0.1,0.2,0.3\}) \\
(M,\{-0.1,0.1,0.3\}) & (P,\{-0.2,-0.1,0.0\}) & (M,\{0.1,0.4,0.45\}) & (E G,\{-0.05,0.25\})
\end{array}\right]
\end{aligned}
$$

Step 2 Use the DH2TA operator to aggregate value of $\left(l_{i j k}^{p}, \varsigma^{p}\right)(i=1,2,3,4,5, j=1,2,3,4, p=1,2,3$ and $k \in\{0,1,2, \ldots, 6\})$ for all decision maker's evaluation as follow:

$$
\begin{aligned}
& M_{\text {agg }}= \\
& {\left[\begin{array}{cccc}
(M,\{-0.3,-0.1\}) & (V G,\{-0.1,0.1,0.2\}) & (P,\{0.2\}) & (P,\{0.1\}) \\
(P,\{-0.1,0.0,0.1,0.2,0.4\}) & (P,\{0.0,0.2\}) & (G,\{-0.2,0.1,0.3\}) & (G,\{-0.2,-0.1\}) \\
(M,\{0.1\}) & (M,\{-0.05,0.2\}) & (G,\{0.1,0.25\}) & (V P,\{-0.3,-0.2,0.0\}) \\
(V G,\{0.0,0.2\}) & (M,\{0.0,0.2,0.25,0.3\}) & (P,\{-0.3,0.1,0.2\}) & (M,\{-0.25,-0.1,0.1\}) \\
(G,\{-0.1,0.1\}) & (P,\{-0.1,0.0) & (P,\{-0.2,-0.1,0.1\}) & (V G,\{-0.05,0.0\})
\end{array}\right]}
\end{aligned}
$$


Step 3. To find the fuzzy measures for attributes of $Y=\left\{Y_{1}\right.$ (Price), $Y_{2}$ (Quality), $Y_{3}$ (Delivery time), $Y_{4}($ Financial status of company) $\}$ and parameter $\lambda$. Let $\alpha\left(Y_{1}\right)=0.3, \alpha\left(Y_{2}\right)=0.25, \alpha\left(Y_{3}\right)=0.15$ and $\alpha\left(Y_{4}\right)=$ 0.29. Then by equation $2.3, \lambda=0.00277$ and by equation $2.2, \alpha\left(Y_{1}, Y_{2}\right)=0.5502, \alpha\left(Y_{1}, Y_{3}\right)=0.4501$, $\alpha\left(Y_{1}, Y_{4}\right)=0.5902, \alpha\left(Y_{2}, Y_{3}\right)=0.4001, \alpha\left(Y_{2}, Y_{4}\right)=0.5402, \alpha\left(Y_{3}, Y_{4}\right)=0.4401, \alpha\left(Y_{1}, Y_{2}, Y_{3}\right)=$ 0.7004, $\alpha\left(Y_{1}, Y_{2}, Y_{4}\right)=0.8406, \alpha\left(Y_{1}, Y_{3}, Y_{4}\right)=0.7405, \alpha\left(Y_{2}, Y_{3}, Y_{4}\right)=0.6904, \alpha\left(Y_{1}, Y_{2}, Y_{3}, Y_{4}\right)=1$.

To find $D C H 2 T A$ aggregate value for the following elements, firstly we use $w_{i j}=\alpha\left(H_{i \sigma(j)}\right)-\alpha\left(H_{i \sigma(j-1)}\right)$ weight for each element.

$$
\begin{aligned}
& \left(l_{1 \sigma(1)}, \varsigma_{1 \sigma(1)}\right)=(V G,\{-0.1,0.1,0.2\}) \\
& \left(l_{1 \sigma(2)}, \varsigma_{1 \sigma(2)}\right)=(M,\{-0.3,-0.1\}) \\
& \left(l_{1 \sigma(3)}, \varsigma_{1 \sigma(3)}\right)=(P,\{0.2\}) \\
& \left(l_{1 \sigma(4)}, \varsigma_{1 \sigma(4)}\right)=(P,\{0.1\})
\end{aligned}
$$

As $H_{1 \sigma(1)}=\left\{Y_{2}\right\}, H_{1 \sigma(2)}=\left\{Y_{1}, Y_{2}\right\}$ and $H_{1 \sigma(3)}=\left\{Y_{1}, Y_{2}, Y_{3}\right\}, H_{1 \sigma(4)}=\left\{Y_{1}, Y_{2}, Y_{3}, Y_{4}\right\}$ we can get $w_{11}=0.25, w_{12}=0.3002$ and $w_{13}=0.1502, w_{14}=0.2996$.

$$
\begin{aligned}
(l, \varsigma)_{1} & =\operatorname{DCH} 2 T A_{\alpha}\left(\left(l_{1 \sigma(1)}, \varsigma_{1 \sigma(1)}\right),\left(l_{1 \sigma(2)}, \varsigma_{1 \sigma(2)}\right),\left(l_{1 \sigma(3)}, \varsigma_{1 \sigma(3)}\right),\left(l_{1 \sigma(4)}, \varsigma_{1 \sigma(4)}\right)\right) \\
& =(M,\{-0.0700,-0.0537,-0.0163,0.2138\}) \\
S\left((l, \varsigma)_{1}\right) & =0.0184
\end{aligned}
$$

Similarly, find the values of $(l, \varsigma)_{2},(l, \varsigma)_{3},(l, \varsigma)_{4}$ and $(l, \varsigma)_{5}$ are

$$
\begin{aligned}
(l, \varsigma)_{2} & =\operatorname{DCH} 2 T A_{\alpha}\left(\left(l_{1 \sigma(1)}, \varsigma_{1 \sigma(1)}\right),\left(l_{1 \sigma(2)}, \varsigma_{1 \sigma(2)}\right),\left(l_{1 \sigma(3)}, \varsigma_{1 \sigma(3)}\right),\left(l_{1 \sigma(4)}, \varsigma_{1 \sigma(4)}\right)\right) \\
(l, \varsigma)_{2} & =(M,\{-0.1967,-0.146,-0.0921\}) \\
S\left((l, \varsigma)_{2}\right) & =-0.1449 \\
(l, \varsigma)_{3} & =D C H 2 T A_{\alpha}\left(\left(l_{1 \sigma(1)}, \varsigma_{1 \sigma(1)}\right),\left(l_{1 \sigma(2)}, \varsigma_{1 \sigma(2)}\right),\left(l_{1 \sigma(3)}, \varsigma_{1 \sigma(3)}\right),\left(l_{1 \sigma(4)}, \varsigma_{1 \sigma(4)}\right)\right) \\
(l, \varsigma)_{3} & =(M,\{-0.0674,-0.0072,0.0924,0.2177\}) \\
S\left((l, \varsigma)_{3}\right) & =0.0589 \\
(l, \varsigma)_{4}= & D C H 2 T A_{\alpha}\left(\left(l_{1 \sigma(1)}, \varsigma_{1 \sigma(1)}\right),\left(l_{1 \sigma(2)}, \varsigma_{1 \sigma(2)}\right),\left(l_{1 \sigma(3)}, \varsigma_{1 \sigma(3)}\right),\left(l_{1 \sigma(4)}, \varsigma_{1 \sigma(4)}\right)\right) \\
(l, \varsigma)_{4}= & (M,\{0.0462,0.0654,0.0955\}) \text { and } S\left((l, \varsigma)_{4}\right)=0.0690
\end{aligned}
$$


and

$$
\begin{aligned}
(l, \varsigma)_{5} & =\operatorname{DCH} 2 T A_{\alpha}\left(\left(l_{1 \sigma(1)}, \varsigma_{1 \sigma(1)}\right),\left(l_{1 \sigma(2)}, \varsigma_{1 \sigma(2)}\right),\left(l_{1 \sigma(3)}, \varsigma_{1 \sigma(3)}\right),\left(l_{1 \sigma(4)}, \varsigma_{1 \sigma(4)}\right)\right) \\
(l, \varsigma)_{5} & =(M,\{-0.3002,-0.2846,-0.2118,-0.0477,-0.0380,-0.0163\}) \\
S\left((l, \varsigma)_{5}\right) & =-0.1498
\end{aligned}
$$

As by definition $3.2(l, \varsigma)_{4}>(l, \varsigma)_{3}>(l, \varsigma)_{1}>(l, \varsigma)_{2}>(l, \varsigma)_{5}$, hence $s_{4} \succ s_{3} \succ s_{1} \succ s_{2} \succ s_{5}$. Therefore, the most suitable supplier's option is $s_{4}$, second, third and four position suppliers are $s_{3}, s_{1}$ and $s_{2}$ respectively, while the worst suppliers option is $s_{5}$.

\section{Discussion AND CONCLUSiON}

Herrera and Martínez [22], discussed a symbolic model and name it 2-tuple linguistic representation model. The 2-tuple linguistic model use words toward processing without loss of any information. In their proposed 2-tuple model, the linguistic term sets were consistent and symmetrically distributed. In view of the Herrera and Martínez [22], the following models have been considered afterward:

- Wang and Hao model [59],

- Herrera et al. model [24],

- Numerical scale model [15].

In each of these models, linguistic term sets examined consistently and symmetrically scattered. Moreover, the symbolic proportions over linguistic terms are precise qualities, and just a single linguistic term set is considered for translation of these qualities. But these models did not address where hesitation occurs between the translation of arguments. Beg and Rashid [5] introduced the concept of hesitant 2-tuple linguistic model to merge Herrera and Martínez's [22], 2-tuple linguistic model with Torra's [55], hesitant fuzzy set. Hesitant 2-tuple linguistic model is very helpful for the situation where decision maker may hesitant to pick a possible value of translation for a linguistic term as it will not cause any loss of information in the process. Beg and Rashid [5] used hesitant 2-tuple linguistic model for the situation where the attributes in the decision making problem are evaluated by hesitant 2-tuple linguistic arguments and they used TOPSIS technique to illustrate hesitant 2-tuple linguistic model's efficiency and feasibility in real-world decision making applications. As TOPSIS technique use maximum and minimum distance or similarity from all terms provide the best option accordingly. Some time the resultant value did not reflect the true picture and fail to find the best result over the argument. Particularly, where we have an interrelation between the arguments. Choquet integral [11] is the best choice where interrelationship is required.

In today globalization era, choice of a suitable supplier for the business in the sense of supply chain management has become a key strategic consideration. But due to natural human hesitation, incomplete supplier information and performances and market uncertainty, a supplier selection process has become more 
complicated. Due to this, it is difficult for decision makers to express their conclusion on the suppliers with exact and crisp values and the evaluations are often expressed in linguistic terms. In such circumstances fuzzy set theory is a very appropriate tool to deal with this kind of problems. In this paper, we have observed a situation that the attributes within the selection for decision making problems are interactive or interdependent and analyze the values in the form of 2 tuple hesitant linguistic arguments. By utilizing the Choquet integral, we have developed DH2TA and DCH2TA aggregation operators. The properties of both operators are studied, such as commutativity, boundedness and monotonicity. We proved that $D H 2 T A$ operator is an idempotent operator. We also utilized DCH2TA operator to the more than one attribute group decision making problems for hesitant 2-tuple linguistic understanding and proposed a method for group decision making problems. An illustrative example has been given to demonstrate the proposed decision making approach. We observe that $D C H 2 T A$ is suitable for conditions where decision making problems are interdependent. The operator $D C H 2 T A$ has the properties to reduce hesitation in aggregated value of hesitant 2-tuple linguistic elements.

In real decision making problem, there involve the interrelationships between the arguments. Often Bonfeeroni mean operators (BM) [7] and Muirhead mean operators (MM) [46] used as the tools where interrelationships between arguments exist. As we observed that diminishing operational laws have the ability to reduce hesitation in resultant argument. In future, we will use this capability of diminishing operational laws and will purpose BM and MM for hesitant 2-tuple linguistic model.

Conflict of interest. The authors declare that they have no conflict of interest.

\section{REFERENCES}

[1] R. D. Andrés, M. Espinilla and L. Martínez, An extended hierarchical linguistic model for managing integral evaluation, Int. J. Comput. Intell. Syst. 3 (4) (2010), 486-500.

[2] R. D. Andrés, J.L. García-Lapresta and L. Martínez, A multi-granular linguistic model for management decision-making in performance appraisal, Soft Computing, 14 (1) (2010), 21-34.

[3] C. Araz and I. Ozkarahan, Supplier evaluation and management system for strategic sourcing based on a new multicriteria sorting procedure, Int. J. Production Economics, 106 (2) (2007), 585-606.

[4] I. Beg and T. Rashid, Multi-criteria of bike purchasing using fuzzy choquet integral, J. Fuzzy Math. 22 (3)(2014), 677-694.

[5] I. Beg and T. Rashid, Hesitant 2-tuple linguistic information in multiple attributes group decision making, J. Intell. Fuzzy Syst. 30 (2016), 109-116.

[6] I. Beg and T. Rashid, Modelling uncertainties in multi-criteria decision making using distance measure and TOPSIS for hesitant fuzzy sets, J. Artif. Intell. Soft Comput. Res. 7(2)(2017), 103-109.

[7] C. Bonferroni, Sulle medie multiple di potenze, Bolletino Matematica Italiana, 5 (1950), 267-270.

[8] C. T. Chen, C. T. Lin, and S. F. Huang, A fuzzy approach for supplier evaluation and selection in supply chain management, Int. J. Production Economics, 102 (2) (2006), 289-301.

[9] C.T. Chen, P. F. Pai and W. Z. Hung, An integrated methodology using linguistic promethee and maximum deviation method for third-party logistics supplier selection, Int. J. Comput. Intell. Syst. 3 (4) (2010), 438-451. 
[10] Y. Chen, X. Zeng M. Happiette, P. Bruniaux, R. Ng and W. Yu, Optimisation of garment design using fuzzy logic and sensory evaluation techniques, Eng. Appl. Artif. Intell. 22 (2) (2009), 272-282.

[11] G. Choquet, Theory of capacities. Ann. Inst. Fourier, 5 (1953), 131-295.

[12] S. Y. Chou and Y. H. Chang, A decision support system for supplier selection based on a strategy-aligned fuzzy SMART approach, Expert Syst. Appl. 34 (4) (2008), 2241-2253.

[13] R. Degani and G. Bortolan, The problem of linguistic approximation in clinical decision making, Int. J. Approx. Reason. 2 (1988), 143-162.

[14] M. Delgado, J.L. Verdegay and M.A. Vila, On aggregation operations of linguistic labels, Int. J. Intell. Syst. 8 (3) (1993), $351-370$.

[15] Y.C. Dong, C.C. Li and F. Herrera, An optimization-based approach to adjusting the unbalanced linguistic preference relations to obtain a required consistency level, Inf. Sci. 292 (2015), 27-38.

[16] D. Dubois and H. Prade, Fuzzy Sets and Systems: Theory and Applications, Kluwer Academic, New York, 1980.

[17] C. X. Feng, J. Wang, and J. S. Wang, An optimization model for concurrent selection of tolerances and suppliers, Comput. Ind. Eng. 40 (1-2) (2001), 15-33.

[18] N. Fenton and W. Wang, Risk and confidence analysis for fuzzy multicriteria decision making, Knowl.based Syst. 19 (6) (2006), 430-437.

[19] J.L. García-Lapresta, B. Llamazares and M. Martínez-Panero, A social choice analysis of the Borda rule in a general linguistic framework, Int. J. Comput. Intell. Syst. 3 (4) (2010), 501-513.

[20] R. E. Gregory, Source selection: a matrix approach, J. Purchas. Mater. Manag. 22 (2) (1986), 24-29.

[21] S. H. Ha and R. Krishnan, A hybrid approach to supplier selection for the maintenance of a competitive supply chain, Expert Syst. Appl. 34 (2) (2008), 1303-1311.

[22] F. Herrera and L. Martínez, A 2-tuple fuzzy linguistic representation model for computing with words, IEEE Trans. Fuzzy Syst. 8 (2000), 746-752.

[23] F. Herrera and L. Martínez, The 2-tuple linguistic computational model advantages of its linguistic description, accuracy and consistency, Int. J. Uncertain. Fuzziness Knowl.-Based Syst. 9 (2001), 33-48.

[24] F. Herrera, E. Herrera-Viedma and L. Martínez, A fuzzy linguistic methodology to deal with unbalanced linguistic term sets, IEEE Trans. Fuzzy Syst. 16(2) (2008), 354-370.

[25] E. Herrera-Viedma, A.G. López-Herrera, M. Luque and C. Porcel, A fuzzy linguistic IRS model based on a 2-tuple fuzzy linguistic approach, Int. J. Uncertain. Fuzziness Knowl.-Based Syst. 15 (2) (2007), 225-250.

[26] V.N. Huynh and Y. Nakamori, A satisfactory-oriented approach to multi-expert decision-making under linguistic assessments, IEEE Trans. Syst. Man Cybern. 35 (2) (2005), 184-196.

[27] H. Ishibuchi, T. Nakashima and M. Nii, Classification and Modeling with Linguistic Information Granules: Advanced Approaches to Linguistic Data Mining, Springer, Berlin, 2004.

[28] Y.P. Jiang and Z.P. Fan, Property analysis of the aggregation operators for 2-tuple linguistic information, Control Decision, 18(6) (2003), 754-757.

[29] D.K. Joshi, I. Beg and S. Kumar, Hesitant probabilistic fuzzy linguistic sets with applications in multi-criteria group decision making problems, Mathematics 6(4)(2018), Article ID 47.

[30] A. Khalid and I. Beg, Incomplete hesitant fuzzy preference relations in group decision making, Int. J. Fuzzy Syst. $19(3)(2017), 637-645$.

[31] G.J. Klir and B. Yuan, Fuzzy Sets and Fuzzy Logic: Theory and Applications, Prentice-Hall PTR, 1995. 
[32] C. Labreuche and M. Grabisch, Generalized Choquet-like aggregation functions for handling bipolar scales, Eur. J. Oper. Res. 172 (3) (2006), 931-955.

[33] J. Lawry, A framework for linguistic modelling, Artif. Intell. 155 (1-2) (2004), 1-39.

[34] C. C. Li, Y. Donga, F. Herrera, E. H. Viedm and L. Martínez, Personalized individual semantics in computing with words for supporting linguistic group decision making. An application on consensus reaching, Inf. Fusion, 33 (2017), 29-40.

[35] J. Lu, G. Zhang and F. Wu, Team situation awareness using web-based fuzzy group decision support systems, Int. J. Comput. Intell. Syst. 1 (1) (2008), 51-60.

[36] J. Lu, Y. Zhu, X. Zeng, L. Koehl, J. Ma and G. Zhang, A linguistic multi-criteria group decision support system for fabric hand evaluation, Fuzzy Optim. Decis. Mak. 8 (4) (2009), 395-413.

[37] O. Martin and G.J. Klir, On the problem of retranslation in computing with perceptions, Int. J. General Syst. 35 (6) (2006), 655-674.

[38] L. Martínez, D. Ruan, F. Herrera, E. Herrera-Viedma and P.P. Wang, Linguistic decision making: tools and applications, Inf. Sci. 179 (14) (2009), 2297-2298.

[39] L. Martínez, J. Liu, D. Ruan and J.B. Yang, Dealing with heterogeneous information in engineering evaluation processes, Inf. Sci. 177 (7) (2007), 1533-1542.

[40] L. Martínez, J. Liu, J. B. Yang and F. Herrera, A multigranular hierarchical linguistic model for design evaluation based on safety and cost analysis, Int. J. Intell. Syst. 20 (12) (2005), 1161-1194.

[41] L. Martínez, R. M. Rodríguez and F. Herrera, 2-tuple Linguistic Model Computing with words in Decision Making, Springer (2015).

[42] J.M. Mendel and D. Wu, Perceptual Computing: Aiding People in Making Subjective Judgments, IEEE-Wiley, (2010).

[43] R. Mesiar and A. Kolesarova, On the fuzzy set theory and aggregation functions: Histor and some recent advances, Iran. J. Fuzzy Syst. in press.

[44] W.R.W. Mohd and L. Abdullah, Aggregation methods in group decision making: A decade survey, Informatica 41 (2017), $71-86$.

[45] G.A. Miller, The magical number seven, plus or minus two: some limits on our capacity of processing information, Psychol. Rev. 63 (1956), 81-97.

[46] R. F. Muirhead, Some methods applicable to identities and inequalities of symmetric algebraic functions of $n$ letters, Proc. Edinburgh Math. Soc. 21(3) (1902), 144-162.

[47] R. Narasimhan, An analytic approach to supplier selection, J. Purchas. Supply Manag. 1 (1983), 27-32.

[48] R. L. Nydick and R. P. Hill, Using the Analytic Hierarchy Process to structure the supplier selection procedure, Int. J. Purchas. Mat. Manag. 28 (2) (1992), 31-36.

[49] L. Pérez-Domínguez, L. Rodríguez-Picón, A. Alvarado-Iniesta, D.L. Cruz and Z.S. Xu, MOORA under Pythagorean fuzzy set for multiple criteria decision making, Complexity, 2018 (2008), Article ID 2602376, 10 pages

[50] I. Saad, S. Hammadi, M. Benrejeb and P. Borne, Choquet integral for criteria aggregation in the flexible job-shop scheduling problems, Math. Comput. Simul. 76 (2008), 447-462.

[51] H. Shi, H. C. Liu, P. Li, X. and G. Xu, An integrated decision making approach for assessing healthcare waste treatment technologies from a multiple stakeholder, Waste Manag. 59 (2017), 508-517.

[52] W. R. Soukup, Supplier selection strategies, J. Purchas. Mat. Manag. 23 (3) (1987), 7-12.

[53] C. Q. Tan and X. H. Chen, Intuitionistic fuzzy Choquet integral operator for multi-criteira decision making, Expert Syst. Appl. 37 (2010), 149-157.

[54] Thompson, Vendor prole analysis, J. Purchas. Mat. Manag. 26 (1) (1990), 11-18. 
[55] V. Torra, Hesitant fuzzy sets, Int. J. Intell. Syst. 25 (2010), 529-539.

[56] R. J. Vokurka, J. Choobineh, and L. Vadi, A prototype expert system for the evaluation and selection of potential suppliers, Int. J. Oper. Prod. Manag. 16 (12) (1996), 106-127.

[57] P. Wakker, Additive representations of preferences, Kluwer Academic Publishers, (1999).

[58] Z. Wang and G. Klir, Fuzzy measure theory, New York: Plenum press, (1992).

[59] J.H. Wang and J.Y. Hao, A new version of 2-tuple fuzzy linguistic representation model for computing with words, IEEE Trans. Fuzzy Syst. 14(3) (2006), 435-445.

[60] G.W. Wei, Method for two-tuple linguistic group decision making based on the ET-WG and ET-OWG operators, Expert Syst. Appl. 37 (2010), 7895-7900.

[61] M.M. Xia, Z.S. Xu and B. Zhu, Geometric Bonferroni means with their application in multi-criteria decision making, Knowl. based Syst. 40 (2013), 88-100.

[62] Z.S. Xu, A method based on linguistic aggregation operators for group decision making with linguistic preference relations, Inf. Sci. 166(1-4) (2004), 19-30.

[63] Z.S. Xu, EOWA and EOWG operators for aggregating linguistic labels based on linguistic preference relations, Int. J. Uncertain. Fuzziness Knowl.-Based Syst. 12 (2004), 791-810.

[64] Z. Xu, S. Shang, W. Qian and W. Shu, A method for fuzzy risk analysis based on the new similarity of trapezoidal fuzzy numbers, Expert Syst. Appl. 37 (3) (2010), 1920-1927.

[65] R.R. Yager, A new methodology for ordinal multi objective decisions based on fuzzy sets, Decision Sci. 12 (1981), 589-600.

[66] R.R. Yager, Computing with Words and Information/Intelligent Systems 2: Applications, Chapter Approximate Reasoning as a Basis for Computing with Words, Physica Verlag, (1999), 50-77.

[67] R.R. Yager, Induced aggregation operators, Fuzzy Sets Syst. 137 (2003), 59-69.

[68] R.R. Yager, On the retranslation process in Zadeh's paradigm of computing with words, IEEE Transactions on Systems, Man, and Cybernetics - Part B: Cybernetics, 34 (2) (2004), 1184-1195.

[69] R.R. Yager, OWA aggregation of intuitionistic fuzzy sets, Int. J. General Syst. 38 (6) (2009), 617-641.

[70] W. Yang and Z. Chen, New aggregation operators based on the Choquet integral and 2-tuple linguistic information, Expert Syst. Appl. 39 (2012), 2662-2668

[71] L. Zadeh, Fuzzy sets, Inf. Control, 8 (1965), 338-353.

[72] L. Zadeh, The concept of a linguistic variable and its application to approximate reasoning, Part III, Inf. Sci. 9 (1) (1975), $43-80$.

[73] Y. Zhang and Z.P. Fan, An approach to linguistic multiple attribute decision-making with linguistic information based on ELOWA operator, Syst. Eng. 24(12) (2006), 324-339.

[74] B. Zhu, Z. S. Xu and M. M. Xia, Hesitant fuzzy geometric Bonferroni means, Inf. Sci. 205 (2012), 72-85. 